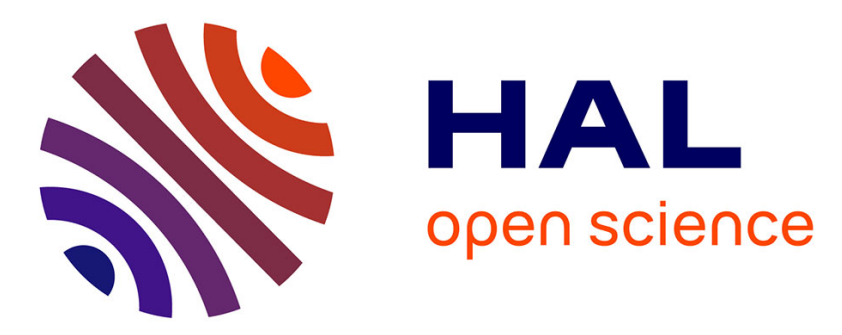

\title{
Elliptic PDEs with distributional drift and backward SDEs driven by a càdlàg martingale with random terminal time
}

\author{
Francesco Russo, Lukas Wurzer
}

\section{To cite this version:}

Francesco Russo, Lukas Wurzer. Elliptic PDEs with distributional drift and backward SDEs driven by a càdlàg martingale with random terminal time. Stochastics and Dynamics, 2017, 17, pp.1750030 . 10.1142/S0219493717500307 . hal-01023176v2

\section{HAL Id: hal-01023176 \\ https://hal.inria.fr/hal-01023176v2}

Submitted on 2 Jun 2015

HAL is a multi-disciplinary open access archive for the deposit and dissemination of scientific research documents, whether they are published or not. The documents may come from teaching and research institutions in France or abroad, or from public or private research centers.
L'archive ouverte pluridisciplinaire HAL, est destinée au dépôt et à la diffusion de documents scientifiques de niveau recherche, publiés ou non, émanant des établissements d'enseignement et de recherche français ou étrangers, des laboratoires publics ou privés. 


\title{
Elliptic PDEs with distributional drift and backward SDEs driven by a càdlàg martingale with random terminal time
}

\author{
Francesco Russo $^{1}$ and Lukas Wurzer ${ }^{2}$ \\ ${ }^{1}$ Ecole Nationale Supérieure des Techniques Avancées, ENSTA \\ ParisTech, Unité de Mathématiques appliquées \\ ${ }^{2}$ University of Innsbruck, Institute for Basic Sciences in Engineering \\ Sciences, Unit for Engineering Mathematics
}

June 2nd, 2015

\begin{abstract}
We introduce a generalized notion of semilinear elliptic partial differential equations where the corresponding second order partial differential operator $L$ has a generalized drift. We investigate existence and uniqueness of generalized solutions of class $C^{1}$. The generator $L$ is associated with a Markov process $X$ which is the solution of a stochastic differential equation with distributional drift. If the semilinear PDE admits boundary conditions, its solution is naturally associated with a backward stochastic differential equation (BSDE) with random terminal time, where the forward process is $X$. Since $X$ is a weak solution of the forward SDE, the BSDE appears naturally to be driven by a martingale. In the paper we also discuss the uniqueness of solutions of a BSDE with random terminal time when the driving process is a general càdlàg martingale.
\end{abstract}

KEY WORDS AND PHRASES: Backward stochastic differential equations; random terminal time; martingale problem; distributional drift; elliptic partial differential equations.

MSC 2010: 60H10; 60H30; 35H99.

\section{Introduction}

The paper involves three essential areas of study. 
1. Elliptic semilinear PDEs with distributional drift.

2. Backward stochastic differential equations (BSDEs) driven by càdlàg martingales with terminal condition at random terminal time.

3. The representation of solutions of the above mentioned BSDEs through solutions of PDEs.

We consider a differential equation of the type

$$
L u=F\left(x, u, u^{\prime}\right),
$$

on $[0,1]$ with boundary conditions, where $L$ is the generator of a one-dimensional stochastic differential equation of the type $L g=\frac{\sigma^{2}}{2} g^{\prime \prime}+\beta^{\prime} g^{\prime}$, with $\sigma, \beta$ being real continuous functions and $\sigma$ is strictly positive. In general, (1.1) is a semilinear PDE, which reduces to an ODE in the case of one dimension considered here. The drift $\beta^{\prime}$ is the derivative of a continuous function $\beta$, in general a distribution. A typical example of such $\beta$ is the path of a fixed continuous process. $F$ is a continuous real function defined on $[0,1] \times \mathbb{R}^{2}$. When $F$ does not depend on $u$ and $u^{\prime}$, and $x$ varies on the real line, (1.1) was introduced in $[14,15]$, via the notion of $C^{1}$-solutions which appear as limit of solutions of elliptic problems with regularized coefficients. Indeed $[14,15]$ investigated the case of initial conditions.

One-dimensional stochastic differential equations with distributional drift were examined by several authors, see [14, 15, 2, 23] and references therein, with a recent contribution by [19]. Such an equation appears formally as

$$
d X_{t}=\beta^{\prime}\left(X_{t}\right) d t+\sigma\left(X_{t}\right) d W_{t}
$$

More recently some contributions also appeared in the multidimensional case, see [1], when the drift is a Kato class measure and in [13] for other type of time dependent drifts.

A motivation for studying the mentioned type of equations comes from the literature of random media. A special case of equation (1.2) with $a=1$ and $\beta$ being the continuous function $\beta$ was considered by several authors, see e.g. $[21,20,17,26]$, in particular in relation with long time behavior, without defining the stochastic analysis framework. In that case, the solution $X$ of (1.2) is the so called Brox diffusion. The discrete version of this is the random walk in random environment. In that case the solution $X$ describes the motion of a particle in an irregular medium: the velocity of the medium $\beta^{\prime}$ can be for instance the realization of a Gaussian white noise but the noise could be also of other nature. In particular $\beta$ is often a (possibly fractional) Brownian path, but it could be the path any continuous process.

This paper is devoted to the following main objectives.

1. We study existence and uniqueness of a solution $u$ of the semilinear equation (1.1) with prescribed initial conditions for $u(0)$ and $u^{\prime}(0)$, see Proposition 3.6.

2. We show that the initial value problem allows to provide a solution to the boundary value problem on $[0,1]$ for $(1.1)$, see Proposition 3.12 . 
3. We explore several assumptions on $F$ which provide existence and/or uniqueness of solutions to the boundary value problem, see Corollary 3.11 and Propositions 3.12 and 3.14 .

4. We study the uniqueness of solutions of BSDEs driven by a càdlàg martingale $M$ such that $\langle M\rangle$ is continuous, see Theorem 5.3.

5. We show that a solution of the PDE (1.1) with Dirichlet boundary conditions on $[0,1]$ generates a solution to a special forward BSDE (see Theorem 6.2) with terminal condition at the random time $\tau$, where $\tau$ is the exit time from $[0,1]$ of a solution $X$ of an SDE with distributional drift.

6. Those solutions which are associated with (1.1) are the unique solutions of the corresponding BSDE (in some reasonable class) whenever $F$ fulfills in particular some strict monotonicity condition in the second variable, i. e. (3.18) holds.

7. We illustrate situations where the BSDE admits no uniqueness in a reasonable class but the probabilistic representation still holds.

As we mentioned, a significant object of study is a backward SDE with random terminal time, which was studied and introduced by [11] when the driving martingale is a Brownian motion. BSDEs driven by a càdlàg martingale with fixed time terminal time were studied in $[7,12,9,6]$.

The paper is organized as follows. After the introduction in Section 2, we recall some preliminaries about linear elliptic differential equations with initial condition and the notion of martingale problem related to an SDE with distributional drift. In Section 3, we discuss existence and uniqueness of solutions to (1.1), in Section 4 we discuss the first exit time properties of a solution to equation (1.2). In Section 5 we investigate uniqueness for BSDEs with random terminal condition with related probabilistic representation. Finally Section 6 shows how a solution to (1.1) generates a solution to a special BSDE with terminal condition at random time.

\section{Preliminaries}

\subsection{The linear elliptic PDE with distributional drift}

If $I$ is a real open interval, then $C^{0}(I)$ will be the space of continuous functions on $I$ endowed with the topology of uniform convergence on compacts. For $k \geq 1, C^{k}(I)$ will be the space of $k$-times continuously differentiable functions on $I$, equipped with the topology of uniform convergence of the first $k$ derivatives. If $I=\mathbb{R}, k \geq 0$, then we will simply write $C^{k}$ instead of $C^{k}(\mathbb{R})$. If $I=[a, b]$ with $-\infty<a<b<+\infty$, then $u: I \rightarrow \mathbb{R}$ is said to be of class $C^{1}([a, b])$ if it is of class $C^{1}(] a, b[)$ and if the derivative extends continuously to $[a, b]$.

In this section we introduce the "generator" $L$ of our diffusion with distributional drift adopting the notations and conventions of $[14,15]$. 
Let $\sigma, \beta \in C^{0}$ such that $\sigma>0$. We consider formally a differential operator of the following type [14, section 2]:

$$
L g=\frac{\sigma^{2}}{2} g^{\prime \prime}+\beta^{\prime} g^{\prime}
$$

By a mollifier, we intend a function $\Phi$ belonging to the Schwartz space $\mathcal{S}(\mathbb{R})$ with $\int \Phi(x) d x=1$. We denote

$$
\Phi_{n}(x):=n \Phi(n x), \quad \sigma_{n}^{2}:=\sigma^{2} * \Phi_{n}, \quad \beta_{n}:=\beta * \Phi_{n} .
$$

We then consider

$$
L_{n} g=\frac{\sigma_{n}^{2}}{2} g^{\prime \prime}+\beta_{n}^{\prime} g^{\prime}
$$

A priori, $\sigma_{n}^{2}, \beta_{n}$ and the operator $L_{n}$ depend on the mollifier $\Phi$.

Definition 2.1. Let $l \in C^{0}$. A function $f \in C^{1}(\mathbb{R})$ is said to be a $C^{1}$-solution to

$$
L f=l,
$$

if, for any mollifier $\Phi$, there are sequences $\left(f_{n}\right)$ in $C^{2},\left(l_{n}\right)$ in $C^{0}$ such that

$$
L_{n} f_{n}=l_{n}, \quad f_{n} \rightarrow f \text { in } C^{1}, \quad l_{n} \rightarrow l \text { in } C^{0} .
$$

The following proposition gives conditions for the existence of a solution $h$ to the homogeneous version of (2.3), see [14, Prop. 2.3].

Proposition 2.2. Let $a \in \mathbb{R}$ be fixed. There is a $C^{1}$-solution to Lh $=0$ such that $h^{\prime}(x) \neq 0$ for every $x \in \mathbb{R}$ if and only if

$$
\Sigma(x):=\lim _{n \rightarrow \infty} 2 \int_{a}^{x} \frac{\beta_{n}^{\prime}}{\sigma_{n}^{2}}(y) d y
$$

exists in $C^{0}$, independently from the mollifier. Moreover, in this case, any $C^{1}$-solution $f$ to $L f=0$ fulfills

$$
f^{\prime}(x)=e^{-\Sigma(x)} f^{\prime}(a), \forall x \in \mathbb{R} .
$$

\section{Remark 2.3.}

1. In particular, this proves the uniqueness of solutions to the problem

$$
L f=l, \quad f \in C^{1}, \quad f(a)=x_{0}, \quad f^{\prime}(a)=x_{1},
$$

for every $l \in C^{0}, x_{0}, x_{1} \in \mathbb{R}$.

2. In most of the cases we will set $a=0$.

In the sequel we will always suppose the existence of $\Sigma$ as in (2.5). We will denote $h: \mathbb{R} \rightarrow \mathbb{R}$ such that $h(0)=0$ and $h^{\prime}=\exp (-\Sigma)$ and $h_{n}: \mathbb{R} \rightarrow \mathbb{R}$ so that $h_{n}=\exp \left(-\Sigma_{n}\right)$ with $\Sigma_{n}=2 \int_{0}^{x} \frac{\beta_{n}^{\prime}}{\sigma_{n}^{2}}(y) d y$. The proposition below is a consequence of [14, Lemma 2.6] and [14, Remark 2.7]. 
Proposition 2.4. Let $a \in \mathbb{R}$ and $l \in C^{0}$ and $x_{0}, x_{1} \in \mathbb{R}$. Then there is a unique $C^{1}$-solution to

$$
\begin{aligned}
L u & =l \\
u(a) & =x_{0}, \quad u^{\prime}(a)=x_{1} .
\end{aligned}
$$

The solution satisfies

$$
u^{\prime}(x)=e^{-\Sigma(x)}\left(2 \int_{a}^{x} e^{\Sigma(y)} \frac{l(y)}{\sigma^{2}(y)} d y+x_{1}\right) .
$$

We will denote by $\mathcal{D}_{L}$ the set of all $f \in C^{1}$ which are $C^{1}$-solutions of $L f=l$ for some $l \in C^{0}$. This defines without ambiguity $L: \mathcal{D}_{L} \rightarrow C^{0}$.

\subsection{Related martingale problem}

For the moment we fix a probability space $(\Omega, \mathcal{G}, \mathbb{P})$. All processes will be considered on the index set $\mathbb{R}_{+}$.

For convenience, we follow the framework of stochastic calculus introduced in [25] and developed in several papers. A survey of that calculus in finite dimension is given in [24]. We will fix a filtration $\mathcal{F}=\left(\mathcal{F}_{t}\right)$ which will fulfill the usual conditions.

The covariation of two continuous processes $X$ and $Y$ is defined as follows. Suppose that

$$
A_{t}:=\lim _{\varepsilon \rightarrow 0+} C_{\varepsilon}(X, Y)_{t}
$$

exists for any $t \in[0, T]$ in probability, where

$$
C_{\varepsilon}(X, Y)_{t}:=\frac{1}{\varepsilon} \int_{0}^{t}\left(X_{s+\varepsilon}-X_{s}\right)\left(Y_{s+\varepsilon}-Y_{s}\right) d s .
$$

We say that $(X, Y)$ admit a covariation if the random function $\left(A_{t}\right)$ admits a (necessarily unique) continuous version, which will be designated by $[X, Y]$. For $[X, X]$ we often shortly write $[X]$. All the covariation processes will be continuous.

Remark 2.5. In [24, Propositions 1, 9 and 11, Remarks 1 and 2] we can find the following.

a) If $[X, X]$ exists, then it is always an increasing process and $X$ is called a finite quadratic variation process. If $[X, X] \equiv 0$, then $X$ is said to be a zero quadratic variation process.

b) Let $X$ and $Y$ be continuous processes such that $[X, Y],[X, X],[Y, Y]$ exist. Then $[X, Y]$ is a bounded variation process. If $f, g \in C^{1}$, then

$$
[f(X), g(Y)]_{t}=\int_{0}^{t} f^{\prime}(X) g^{\prime}(Y) d[X, Y]
$$


c) If $A$ is a zero quadratic variation process and $X$ is a finite quadratic variation process, then $[X, A] \equiv 0$.

d) A bounded variation process is a zero quadratic variation process.

e) If $M$ and $N$ are $\mathcal{F}$-local martingales, then $[M, N]$ is the usual covariation process $\langle M, N\rangle$.

An $\mathcal{F}$-Dirichlet process is the sum of an $\mathcal{F}$-local continuous martingale $M$ and an $\mathcal{F}$-adapted zero quadratic variation process $A$, see $[16,4]$.

Remark 2.6. Let $X=M+A$ be an $\mathcal{F}$-Dirichlet process.

1. Remark 2.5c) and e) together with the bilinearity of the covariation operator imply that $[X]=\langle M\rangle$.

2. If $f \in C^{1}$, then $f(X)=M^{f}+A^{f}$ is an $\mathcal{F}$-Dirichlet process, where

$$
M^{f}=\int_{0}^{\cdot} f^{\prime}\left(X_{s}\right) d M_{s}
$$

and $A^{f}:=f(X)-M^{f}$ has zero quadratic variation. This easily follows from the bilinearity of covariation and Remark 2.5b), c) and e). See also [4] for a similar result and Proposition 17 in [24] for a generalization to weak Dirichlet processes.

Definition 2.7. Given a stopping time $\tau$ and a process $X$, we denote by $X^{\tau}$ the stopped process

$$
X_{t}^{\tau}:=X_{t \wedge \tau}, \quad t \geq 0 .
$$

Remark 2.8. Let $\tau$ be an $\mathcal{F}$-stopping time. If $X$ is an $\mathcal{F}$-semimartingale (resp. $\mathcal{F}$ Dirichlet process), then the stopped processes $X^{\tau}$ is also a semimartingale (resp. $\mathcal{F}$ Dirichlet process).

In the classical theory of Stroock and Varadhan, see e.g. [27], the solutions of martingale problems are probabilities on the canonical space $C^{0}([0, T])$ equipped with its Borel $\sigma$-field and the Wiener measure. Here the meaning is a bit different since the solutions are considered to be processes. For the sequel of the section we fix $x_{0} \in \mathbb{R}$.

Definition 2.9. A process $X$ (defined on some probability space), is said to solve the martingale problem $\operatorname{MP}\left(\sigma, \beta ; x_{0}\right)$ if $X_{0}=x_{0}$ a.s. and

$$
f\left(X_{t}\right)-f\left(x_{0}\right)-\int_{0}^{t} L f\left(X_{s}\right) d s
$$

is a local martingale for any $f \in \mathcal{D}_{L}$. $X$.

In the sequel we will denote by $\mathcal{F}^{X}=\left(\mathcal{F}_{t}^{X}\right)$ the canonical filtration associated with 
Definition 2.10. We say that the martingale problem $\operatorname{MP}\left(\sigma, \beta ; x_{0}\right)$ admits uniqueness (in law) if any processes $X_{1}$ and $X_{2}$, defined on some probability space and solving the martingale problem, have the same law.

The proposition below was the object of Proposition 3.13 of [14].

Proposition 2.11. Let $v$ be the unique solution to $L v=1$ in the $C^{1}$-sense such that $v(0)=v^{\prime}(0)=0$. Then there exists a unique (in law) solution the martingale problem $\operatorname{MP}\left(\sigma, \beta ; x_{0}\right)$ if and only if

$$
v(-\infty)=v(+\infty)=+\infty .
$$

In several contexts (see [14]) the solution of the previous martingale problem turns out to be a solution (in the proper sense) of (1.2), but it will not be used in this paper.

Proposition 2.11 implies the following.

Proposition 2.12. The martingale problem $\operatorname{MP}\left(\sigma, \beta ; x_{0}\right)$ admits exactly one solution in law if and only if the function $v: \mathbb{R} \rightarrow \mathbb{R}$ defined by

$$
\begin{aligned}
v(0) & =0 \\
v^{\prime}(x) & =e^{-\Sigma(x)}\left(2 \int_{0}^{x} \frac{1}{\sigma^{2}}(y) d y\right)
\end{aligned}
$$

fulfills

$$
v(-\infty)=v(+\infty)=+\infty
$$

Proof. This follows from Proposition 2.11, Proposition 2.4 and from the fact that $v$ defined in (2.13) is the solution of the problem

$$
L v=1, \quad v(0)=v^{\prime}(0)=0 .
$$

From now on Assumption (2.14) for the function $v$ defined by (2.13) will always be in force. Let then $X$ be a solution to the martingale problem on a suitable probability space and $\mathcal{F}^{X}$ be its canonical filtration.

\section{Remark 2.13.}

i) By Remark 3.3 of [14], choosing $f$ as the identity function, $X$ is an $\mathcal{F}^{X}$-Dirichlet process, whose local martingale part $M^{X}$ verifies

$$
\left[M^{X}\right]_{t}=\int_{0}^{t} \sigma^{2}\left(X_{s}\right) d s .
$$

ii) Consequently by Remark 2.5c) and e) together with the bilinearity of covariation it follows $[X]_{t}=\int_{0}^{t} \sigma^{2}\left(X_{s}\right) d s$. 
Proposition 2.14. Let $X$ be a solution of $\operatorname{MP}\left(\sigma, \beta ; x_{0}\right)$. For every $\varphi \in \mathcal{D}_{L}$ we have

$$
\varphi\left(X_{t}\right)=\varphi\left(X_{0}\right)+\int_{0}^{t} \varphi^{\prime}\left(X_{s}\right) d M_{s}^{X}+\int_{0}^{t}(L \varphi)\left(X_{s}\right) d s .
$$

Proof. By definition of the martingale problem there is an $\mathcal{F}^{X}$-local martingale $M^{\varphi}$ such that

$$
\varphi\left(X_{t}\right)=\varphi\left(X_{0}\right)+M_{t}^{\varphi}+\int_{0}^{t}(L \varphi)\left(X_{s}\right) d s
$$

On the other hand, by Remark 2.13i) and Remark $2.6 \varphi\left(X_{t}\right)$ is an $\mathcal{F}^{X}$-Dirichlet process with decomposition

$$
\varphi\left(X_{t}\right)=\varphi\left(X_{0}\right)+\int_{0}^{t} \varphi^{\prime}\left(X_{s}\right) d M_{s}^{X}+A_{t}^{\varphi}
$$

where $\left[A^{\varphi}\right] \equiv 0$. By the uniqueness of Dirichlet decomposition and the identification of (2.15) and (2.16) the result follows.

\section{The semilinear elliptic PDE with distributional drift and boundary conditions}

In this section we present the deterministic analytical framework that we will need in the paper.

\subsection{The linear case}

We explain here how to transform the study of our initial value problem to a boundary value problem.

Definition 3.1. Let $a, b, A, B \in \mathbb{R}$, such that $-\infty<a<b<\infty$. Additionally, let $g:[a, b] \rightarrow \mathbb{R}$ be continuous. We say that $u:[a, b] \rightarrow \mathbb{R}$ is a solution of the boundary value problem

$$
\left\{\begin{array}{r}
L u=g, \\
u(a)=A, \\
u(b)=B,
\end{array}\right.
$$

if there is a continuous extension $\tilde{g}: \mathbb{R} \rightarrow \mathbb{R}$ of $g$ and a function $\tilde{u} \in \mathcal{D}_{L}$ fulfilling $\left.\tilde{u}\right|_{[a, b]}=u$, such that $\tilde{u}$ is a solution of

$$
L \tilde{u}=\tilde{g},
$$

in the sense of Definition 2.1, and $\tilde{u}(a)=A, \tilde{u}(b)=B$. 
Proposition 3.2. Let $g:[0,1] \rightarrow \mathbb{R}$ be continuous, $A, B \in \mathbb{R}, a=0$ and $b=1$. Then there exists a unique solution $u$ to (3.1), given by

$$
\begin{aligned}
u(x) & =f(x)+\int_{0}^{1} K(x, y) g(y) d y \\
f(x) & :=\frac{B \int_{0}^{x} d y e^{-\Sigma(y)}+A \int_{x}^{1} d y e^{-\Sigma(y)}}{\int_{0}^{1} d y e^{-\Sigma(y)}} \\
K(x, y) & :=\mathbb{1}_{y \leq x} \frac{2 e^{\Sigma(y)}}{\sigma^{2}(y)} \int_{y}^{x} d z e^{-\Sigma(z)}-2 \frac{\int_{0}^{x} d r e^{-\Sigma(r)}}{\int_{0}^{1} d r e^{-\Sigma(r)}} \frac{e^{\Sigma(y)}}{\sigma^{2}(y)} \int_{y}^{1} d z e^{-\Sigma(z)} .
\end{aligned}
$$

Remark 3.3. For every $y \in[0,1], x \mapsto K(x, y)$ is absolutely continuous and $(x, y) \mapsto$ $\partial_{x} K(x, y)$ belongs to $L^{\infty}\left([0,1]^{2}\right)$.

Proof of Proposition 3.2. We start with existence. Let $\tilde{g}$ be a continuous extension of $g$ and $x_{1} \in \mathbb{R}$. Then, by Proposition 2.4, there exists a unique solution $\tilde{u}$ to the problem on the real line,

$$
\begin{aligned}
L \tilde{u}(x) & =\tilde{g}(x), x \in \mathbb{R}, \\
\tilde{u}(0) & =A, \\
\tilde{u}^{\prime}(0) & =x_{1},
\end{aligned}
$$

given by

$$
\tilde{u}(x)=A+\int_{0}^{x} e^{-\Sigma(y)}\left(2 \int_{0}^{y} e^{\Sigma(z)} \frac{\tilde{g}(z)}{\sigma^{2}(z)} d z+x_{1}\right) d y .
$$

We look for $x_{1} \in \mathbb{R}$, so that $\tilde{u}(1)=B$. This gives

$$
\begin{aligned}
B & =A+x_{1} \int_{0}^{1} e^{-\Sigma(y)} d y+2 \int_{0}^{1} d y e^{-\Sigma(y)} \int_{0}^{y} d z e^{\Sigma(z)} \frac{\tilde{g}(z)}{\sigma^{2}(z)}, \\
x_{1} & =\frac{B-A-2 \int_{0}^{1} d z e^{\Sigma(z)} \frac{\tilde{g}(z)}{\sigma^{2}(z)} \int_{z}^{1} d y e^{-\Sigma(y)}}{\int_{0}^{1} e^{-\Sigma(y)} d y} .
\end{aligned}
$$

We insert $x_{1}$ into (3.5) and use the fact that $u=\left.\tilde{u}\right|_{[0,1]}$ and $g=\left.\tilde{g}\right|_{[0,1]}$. This gives (3.3), and we get $u(0)=A$ and $u(1)=B$.

To show uniqueness, let $v^{1}$ and $v^{2}$ be two solutions of (3.1), and set $v=v^{1}-v^{2}$. Then there is $\tilde{v} \in \mathcal{D}_{L}$ with $\left.\tilde{v}\right|_{[0,1]}=v$ and an $\tilde{l} \in C^{0}$ with $\left.\tilde{l}\right|_{[0,1]}=0$, so that

$$
\begin{aligned}
L \tilde{v}(x) & =\tilde{l}, \\
\tilde{v}(0) & =\tilde{v}(1)=0 .
\end{aligned}
$$

We need to show that $v \equiv 0$. By Proposition 2.4 we get

$$
\tilde{v}^{\prime}(x)=e^{-\Sigma(x)}\left(2 \int_{0}^{x} \frac{\tilde{l}(y)}{\sigma^{2}(y)} e^{\Sigma(y)} d y+\tilde{v}^{\prime}(0)\right) \quad \forall x \in \mathbb{R} .
$$


In particular, since $\left.\tilde{l}\right|_{[0,1]}=0$, we get

$$
\tilde{v}^{\prime}(x)=e^{-\Sigma(x)} \tilde{v}^{\prime}(0), \quad \forall x \in[0,1] .
$$

Consequently, for $x \in[0,1]$,

$$
\tilde{v}(x)=\left(\int_{0}^{x} d y e^{-\Sigma(y)}\right) \tilde{v}^{\prime}(0) .
$$

Since $\tilde{v}(1)=0$, it follows $\tilde{v}^{\prime}(0)=0$ and so $v(x)=\tilde{v}(x)=0 \forall x \in[0,1]$.

\subsection{Solution of the semilinear problem on the real line}

We extend here the notion of $C^{1}$-solution to the semilinear case.

Definition 3.4. Let $F: \mathbb{R} \times \mathbb{R}^{2} \rightarrow \mathbb{R}$ be a continuous function. We say that $u \in C^{1}$ is a $C^{1}$-solution (on the real line) of

$$
L u=F\left(x, u, u^{\prime}\right)
$$

if $u$ is a $C^{1}$-solution of $L u=h$, with $h: \mathbb{R} \rightarrow \mathbb{R}$ defined by $h(x)=F\left(x, u(x), u^{\prime}(x)\right)$.

Definition 3.5. Let us consider a function $F: I \times \mathbb{R} \times \mathbb{R} \rightarrow \mathbb{R}$,

1. $(x, y, z) \mapsto F(x, y, z)$ will be called globally Lipschitz with respect to $z$ (resp. $(y, z))$ if $F$ is Lipschitz with respect to $z$ (resp. $(y, z)$ ) uniformly on $x$ varying in $I$ and $y$ in $\mathbb{R}$ (resp. uniformly on $x$ varying in $I$ ). More precisely, $F$ is globally Lipschitz with respect to $z$ if there exists some constant $k$, called the Lipschitz constant for $F$, such that

$$
|F(x, y, z)-F(x, y, \tilde{z})| \leq k|z-\tilde{z}|, \forall x \in I, \forall y, z, \tilde{z} \in \mathbb{R} .
$$

Similarly we speak about the Lipschitz constant $k$ related to a function $F$ which is globally Lipschitz with respect to $(y, z)$.

2. Analogously $F$ will be said to have linear growth with respect to $z$ (resp. $(y, z)$ ) if $F$ has linear growth with respect to $z$ (resp. $(y, z)$ ) uniformly on $x$ varying in $I$ and $y$ in $\mathbb{R}$ (resp. uniformly on $x$ varying in $I$ ). Obvious variants will also be used without further comment.

Proposition 3.6. Suppose that $F: \mathbb{R}^{3} \rightarrow \mathbb{R}$, so that $(x, y, z) \mapsto F(x, y, z)$ restricted to $K \times \mathbb{R}^{2}$, for any compact interval $K$, is Lipschitz with respect to $(y, z)$. Then there is a unique solution of

$$
\begin{aligned}
L u & =F\left(x, u(x), u^{\prime}(x)\right), \quad x \in \mathbb{R}, \\
u(0) & =x_{0}, \\
u^{\prime}(0) & =x_{1} .
\end{aligned}
$$


Proof. By Proposition 2.4, $u: \mathbb{R} \rightarrow \mathbb{R}$ of class $C^{1}$ is a $C^{1}$-solution if and only if

$$
\begin{aligned}
u^{\prime}(x) & =e^{-\Sigma(x)}\left(2 \int_{0}^{x} \frac{e^{\Sigma(y)}}{\sigma^{2}(y)} F\left(y, u(y), u^{\prime}(y)\right) d y+x_{1}\right), \quad \forall x \in \mathbb{R}, \\
u(0) & =x_{0} .
\end{aligned}
$$

We can reduce the well-posedness of (3.9) to the well-posedness of

$$
\begin{aligned}
u^{\prime}(x) & =e^{-\Sigma(x)}\left(2 \int_{0}^{x} \frac{e^{\Sigma(y)}}{\sigma^{2}(y)} F\left(y, u(y), u^{\prime}(y)\right) d y+x_{1}\right), \forall x \in[-N, N], \\
u(0) & =x_{0}
\end{aligned}
$$

for every $N \in \mathbb{N}^{*}:=\mathbb{N}-\{0\}$. In the sequel of the proof, since (3.10) depends on $N$, we will often denote it by $(3.10)(N)$.

Indeed, if $u_{N}$ is a solution of $(3.10)(N)$, then any solution of $(3.10)(N+1)$, restricted to $[-N, N]$ is a solution $(3.10)(N)$. In this way the existence of a solution of (3.9) is equivalent to the existence of a family $\left(u_{N}\right)$ of functions which are respectively solutions of $(3.10)(N)$. In the sequel we fix $N \in \mathbb{N}^{*}$ and we study existence and uniqueness for $(3.10)(N)$, which is an ODE in a compact interval. We consider the map $T: C^{1}([-N, N]) \rightarrow C^{1}([-N, N])$ defined by

$$
\begin{aligned}
T f(0) & =x_{0} \\
(T f)^{\prime}(x) & =e^{-\Sigma(x)}\left(2 \int_{0}^{x} \frac{e^{\Sigma(y)}}{\sigma^{2}(y)} F\left(y, f(y), f^{\prime}(y)\right) d y+x_{1}\right) .
\end{aligned}
$$

Clearly a function $u \in C^{1}([-N, N])$ is a solution of $(3.10)(N)$ if and only if $T u=u$. $C^{1}([-N, N])$ is a Banach space equipped with the norm

$$
\|f\|_{N}=\sup _{|x| \leq N}\left[|f(x)|+\left|f^{\prime}(x)\right|\right] .
$$

The norm $\|\cdot\|_{N}$ is equivalent to

$$
\|f\|_{N, \lambda}=\sup _{|x| \leq N}\left(|f(x)|+\left|f^{\prime}(x)\right|\right) e^{\Sigma(x)-\lambda|x|}
$$

where $\lambda>0$ will be suitably chosen later. It remains to show that $T$ admits a unique fixed point. For this we will show that $T$ is a contraction with respect to $\|\cdot\|_{N, \lambda}$. Let 
$u, v \in C^{1}([-N, N])$. Let us denote by $K / 2$ a Lipschitz constant for $F$. We get

$$
\begin{aligned}
& \left|(T u-T v)^{\prime}(x) e^{\Sigma(x)}\right| \\
& \leq 2\left|\int_{0}^{x}\right| F\left(y, u(y), u^{\prime}(y)\right)-F\left(y, v(y), v^{\prime}(y)\right)\left|\frac{e^{\Sigma(y)}}{\sigma^{2}(y)} d y\right| \\
& \leq K \sup _{|z| \leq N} \frac{1}{\sigma^{2}(z)}\left|\int_{0}^{x}\left(\left|u^{\prime}(y)-v^{\prime}(y)\right|+|u(y)-v(y)|\right) e^{\Sigma(y)} d y\right| \\
& \leq K \sup _{|z| \leq N} \frac{1}{\sigma^{2}(z)}\left|\int_{0}^{x} e^{\lambda|y|} d y\right|\|u-v\|_{N, \lambda} \\
& =K \sup _{|z| \leq N} \frac{1}{\sigma^{2}(z)} \frac{e^{\lambda|x|}-1}{\lambda}\|u-v\|_{N, \lambda} .
\end{aligned}
$$

This implies that, for every $x \in[-N, N]$,

$$
\left|(T u-T v)^{\prime}(x)\right| e^{\Sigma(x)-\lambda|x|} \leq \frac{K}{\lambda} \sup _{|z| \leq N} \frac{1}{\sigma^{2}(z)}\|u-v\|_{N, \lambda} .
$$

On the other hand, since $(T u)(0)=(T v)(0)=x_{0}$ we have

$$
\begin{aligned}
|(T u-T v)(x)| & \leq\left|\int_{0}^{x}\right|(T u-T v)^{\prime}(y)|d y| \\
& =\left|\int_{0}^{x} e^{\Sigma(y)-\lambda|y|}\right|(T u-T v)^{\prime}(y)\left|e^{-\Sigma(y)+\lambda|y|} d y\right| \\
& \leq \sup _{|s| \leq N} e^{-\Sigma(s)} \sup _{|y| \leq N}\left(e^{\Sigma(y)-\lambda|y|}\left|(T u-T v)^{\prime}(y)\right|\right) \frac{e^{\lambda|x|}-1}{\lambda} .
\end{aligned}
$$

Finally, taking into account (3.11), we get

$$
e^{\Sigma(x)-\lambda|x|}|(T u-T v)(x)| \leq \frac{K}{\lambda^{2}} \sup _{|s| \leq N} e^{-\Sigma(s)} \sup _{|y| \leq N} e^{\Sigma(y)} \sup _{|z| \leq N} \frac{1}{\sigma^{2}(z)}\|u-v\|_{N, \lambda} .
$$

Summing up (3.11) and (3.12) we get

$$
\|T u-T v\|_{N} \leq C(\lambda)\|u-v\|_{N, \lambda},
$$

where

$$
C(\lambda)=\frac{K}{\lambda} \sup _{|z| \leq N} \frac{1}{\sigma^{2}(z)}+\frac{K}{\lambda^{2}} \sup _{s \leq N} e^{-\Sigma(s)} \sup _{|y| \leq N} e^{\Sigma(y)} \sup _{|x| \leq N} \frac{1}{\sigma^{2}(x)} .
$$

If $C(\lambda)<1,(3.13)$ has shown that $T$ is a contraction. The condition can be fulfilled by choosing $\lambda$ sufficiently large. 


\subsection{The semi-linear case with boundary conditions}

Definition 3.7. Let

i) $a, b \in \mathbb{R}$, such that $0<a<b<\infty$,

ii) $A, B \in \mathbb{R}$, and

iii) $F:[a, b] \times \mathbb{R}^{2} \rightarrow \mathbb{R}$ be a continuous function.

We say that $u:[a, b] \rightarrow \mathbb{R}$ of class $C^{1}([a, b])$ is a solution of the boundary value problem

$$
\left\{\begin{aligned}
L u(x) & =F\left(x, u, u^{\prime}\right), \\
u(a) & =A, \\
u(b) & =B,
\end{aligned}\right.
$$

if $u$ is a solution of the boundary value problem

$$
\left\{\begin{array}{r}
L u=\ell, \\
u(a)=A, \\
u(b)=B,
\end{array}\right.
$$

in the sense of Definition 3.1 with $\ell:[a, b] \rightarrow \mathbb{R}$ defined by $\ell(x)=F\left(x, u(x), u^{\prime}(x)\right)$.

In Section 5 we will observe that solving (3.14) is strongly related to the problem of solving BSDEs with random terminal time.

Lemma 3.8. Suppose that the assumptions of Definition 3.7 are fulfilled. Then, $u$ is a solution of the boundary value problem

$$
\left\{\begin{aligned}
L u(x) & =F\left(x, u, u^{\prime}\right), \\
u(a) & =A \\
u(b) & =B
\end{aligned}\right.
$$

if and only if the functions $u_{1}, u_{2}:[a, b] \rightarrow \mathbb{R}$, given by

$$
\begin{aligned}
& u_{1}=u, \\
& u_{2}=e^{\Sigma} u^{\prime},
\end{aligned}
$$

belong to $C^{1}([a, b])$ and fulfill

$$
\begin{aligned}
& u_{1}^{\prime}(x)=e^{-\Sigma(x)} u_{2}(x), \\
& u_{2}^{\prime}(x)=2 \frac{e^{\Sigma(x)}}{\sigma^{2}(x)} F\left(x, u_{1}(x), e^{-\Sigma(x)} u_{2}(x)\right), \\
& u_{1}(a)=A \\
& u_{1}(b)=B .
\end{aligned}
$$


Proof. Let $u$ be a solution of the boundary value problem (3.15). This means, by Definition 3.7 , that $u$ is a solution of the boundary value problem

$$
\left\{\begin{aligned}
L u & =\ell \\
u(a) & =A \\
u(b) & =B
\end{aligned}\right.
$$

with

$$
\ell(x)=F\left(x, u(x), u^{\prime}(x)\right),
$$

in the sense of Definition 3.1. By that definition, there are continuous extensions $\tilde{u}$ and $\tilde{\ell}$ such that

$$
\begin{aligned}
\left.\tilde{u}\right|_{[a, b]} & =u, \\
\left.\tilde{\ell}\right|_{[a, b]} & =\ell,
\end{aligned}
$$

and

$$
L \tilde{u}=\tilde{\ell}
$$

in the sense of Definition 2.1. Since $\tilde{u} \in C^{1}$, we can define

$$
x_{a}:=\tilde{u}^{\prime}(a) \text {. }
$$

By Proposition 2.4 it follows that

$$
\tilde{u}^{\prime}(x)=e^{-\Sigma(x)}\left(2 \int_{a}^{x} \frac{e^{\Sigma(y)}}{\sigma^{2}(y)} \tilde{\ell}(y) d y+x_{a}\right), \forall x \in \mathbb{R} .
$$

By setting $\tilde{\ell}_{1}, \tilde{\ell}_{2}, \tilde{u}_{1}, \tilde{u}_{2}: \mathbb{R} \rightarrow \mathbb{R}$ as

$$
\begin{aligned}
& \tilde{\ell}_{1}:=\tilde{u}^{\prime}, \\
& \tilde{\ell}_{2}:=2 \frac{e^{\Sigma}}{\sigma^{2}} \tilde{\ell}, \\
& \tilde{u}_{1}=\tilde{u}, \\
& \tilde{u}_{2}=\tilde{u}^{\prime} e^{\Sigma},
\end{aligned}
$$

it yields that $\tilde{u}^{1}, \tilde{u}^{2}$ belong to $C^{1}$ and

$$
\begin{aligned}
& \tilde{u}_{1}^{\prime}(x)=\tilde{\ell}_{1}(x) \forall x \in \mathbb{R}, \\
& \tilde{u}_{2}^{\prime}(x)=\tilde{\ell}_{2}(x) \forall x \in \mathbb{R}, \\
& \tilde{u}_{1}(a)=A, \\
& \tilde{u}_{1}(b)=B .
\end{aligned}
$$

It follows now that $u_{1}, u_{2} \in C^{1}([a, b], \mathbb{R})$, which are respectively restrictions of $\tilde{u}_{1}, \tilde{u}_{2}$, solve (3.16). 
Concerning the converse, let $\left.u_{1}, u_{2} \in C^{1}([a, b], \mathbb{R}]\right)$, so that (3.16) is fulfilled. We define $\tilde{\ell}_{2}: \mathbb{R} \rightarrow \mathbb{R}$ as

$$
\tilde{\ell}_{2}(x)=2 \frac{e^{\Sigma(x)}}{\sigma^{2}(x)} \tilde{\ell}(x)
$$

where $\tilde{\ell}: \mathbb{R} \rightarrow \mathbb{R}$ is a continuous extension of

$$
\ell(x)=F\left(x, u_{1}(x), u_{2}(x) e^{-\Sigma(x)}\right) .
$$

By (3.16), we have for some $x_{a} \in \mathbb{R}$

$$
u_{2}(x)=2 \int_{a}^{x} \frac{e^{\Sigma(y)}}{\sigma^{2}(y)} \tilde{\ell}(y) d y+x_{a}
$$

for $x \in[a, b]$. We define $\tilde{u}_{2}: \mathbb{R} \rightarrow \mathbb{R}$ as the right-hand side of (3.17) for all $x \in \mathbb{R}$. Clearly $\tilde{u}_{2}$ is a $C^{1}$ extension of $u_{2}$. We also define

$$
\tilde{\ell}_{1}(x)=e^{-\Sigma(x)} \tilde{u}_{2}(x), \quad x \in \mathbb{R} .
$$

(3.16) gives

$$
u_{1}^{\prime}(x)=\tilde{\ell}_{1}(x)=e^{-\Sigma(x)} \tilde{u}_{2}(x), \quad x \in[a, b] .
$$

We define $\tilde{u}_{1}(x)=\int_{a}^{x} \tilde{\ell}_{1}(y) d y+A, x \in \mathbb{R}$. $\tilde{u}_{1}$ is a $C^{1}$ extension of $u_{1}$. Consequently, setting $\tilde{u}=\tilde{u}_{1}$, we get

$$
\begin{aligned}
& \tilde{u}^{\prime}(x)=\tilde{u}_{1}^{\prime}(x)=\tilde{\ell}_{1}(x)=e^{-\Sigma(x)} \tilde{u}_{2}(x)=e^{-\Sigma(x)}\left(2 \int_{a}^{x} \frac{e^{\Sigma(y)}}{\sigma^{2}(y)} \tilde{\ell}(y) d y+x_{a}\right), \\
& \tilde{u}(a)=A
\end{aligned}
$$

taking into account (3.17) and the consideration below it. We define $u:[a, b] \rightarrow \mathbb{R}$ as restriction of $\tilde{u}$ and get

$$
\begin{aligned}
& u(a)=\tilde{u}(a)=A, \\
& u(b)=\tilde{u}(b)=\tilde{u}_{1}(b)=u_{1}(b)=B,
\end{aligned}
$$

by (3.16). By Proposition 2.4, Definition 3.1 and Definition 3.4, $u$ is a solution to the boundary value problem (3.15).

The following result provides uniqueness under some monotonicity conditions.

Proposition 3.9. Let

$$
\begin{aligned}
F:[a, b] \times \mathbb{R}^{2} & \rightarrow \mathbb{R}, \\
(x, y, z) & \mapsto F(x, y, z),
\end{aligned}
$$

be a continuous function fulfilling the following assumptions. 
1. $F$ is non-decreasing in $y$, i. e.

$$
(F(x, y, z)-F(x, \tilde{y}, z))(y-\tilde{y}) \geq 0, \forall y, \tilde{y}, z \in \mathbb{R}, x \in[a, b] .
$$

2. $F$ is globally Lipschitz (with respect to $z$ ).

Then, for any $A, B \in \mathbb{R}$, the boundary value problem

$$
\left\{\begin{aligned}
L u(x) & =F\left(x, u, u^{\prime}\right), \\
u(a) & =A \\
u(b) & =B
\end{aligned}\right.
$$

has at most one $C^{1}$-solution.

Proof. Let $u$ and $v$ in $C^{1}([a, b])$ be two solutions of the boundary value problem (3.19) and define

$$
\begin{aligned}
x_{a} & :=u^{\prime}(a), \\
y_{a} & :=v^{\prime}(a) .
\end{aligned}
$$

Then, by Lemma 3.8, we get

$$
\begin{aligned}
& u(x)=A+\int_{a}^{x} d z e^{-\Sigma(z)}\left(2 \int_{a}^{z} \frac{e^{\Sigma(y)}}{\sigma^{2}(y)} F\left(y, u(y), u^{\prime}(y)\right) d y+x_{a}\right), \quad \forall x \in[a, b], \\
& v(x)=A+\int_{a}^{x} d z e^{-\Sigma(z)}\left(2 \int_{a}^{z} \frac{e^{\Sigma(y)}}{\sigma^{2}(y)} F\left(y, v(y), v^{\prime}(y)\right) d y+y_{a}\right), \quad \forall x \in[a, b], \\
& u(a)=v(a)=A \\
& u(b)=v(b)=B .
\end{aligned}
$$

Indeed, we are interested in the $C^{1}$-function

$$
\begin{aligned}
\phi:[a, b] & \rightarrow \mathbb{R} \\
\phi & =u-v,
\end{aligned}
$$

which fulfills $\phi(a)=\phi(b)=0$. We consider now the $C^{2}$-function $\chi$, given by

$$
\begin{aligned}
\chi(a) & =0 \\
\chi^{\prime}(x) & =e^{\Sigma(x)} \phi^{\prime}(x),
\end{aligned}
$$

and we define

$$
\psi:=\chi^{\prime} \phi
$$

By using (3.21b), the monotonicity and Lipschitz conditions, we get, on $[a, b]$,

$$
\begin{aligned}
\psi^{\prime}=\chi^{\prime \prime} \phi+\chi^{\prime} \phi^{\prime} & \geq \chi^{\prime \prime} \phi=2 \frac{e^{\Sigma}}{\sigma^{2}}\left(F\left(x, u, u^{\prime}\right)-F\left(x, v, v^{\prime}\right)\right)(u-v) \\
& \geq 2 \frac{e^{\Sigma}}{\sigma^{2}}\left(F\left(x, \frac{u+v}{2}, u^{\prime}\right)-F\left(x, \frac{u+v}{2}, v^{\prime}\right)\right)(u-v) \geq-\frac{2 k}{\sigma^{2}}\left|\chi^{\prime} \phi\right|,
\end{aligned}
$$


where $k$ is the Lipschitz constant. So we get the differential inequality

$$
\begin{aligned}
\psi^{\prime}(x) & \geq-\frac{2 k}{\sigma^{2}(x)}|\psi(x)|, x \in[a, b], \\
\psi(a) & =0 \\
\psi(b) & =0 .
\end{aligned}
$$

By some basic properties of differential inequalities [28] we get

$$
\psi(x) \geq 0, x \in[a, b] .
$$

On the other hand,

$$
\int_{a}^{b} \psi(x) e^{-\Sigma(x)} d x=\int_{a}^{b} \phi^{\prime}(x) \phi(x) d x=\left.\frac{\phi^{2}(x)}{2}\right|_{a} ^{b}=0 .
$$

Finally, combining (3.22) and (3.23) leads to

$$
\psi(x)=0, \forall x \in[a, b] .
$$

By definition of $\psi$ it follows that $\left(\phi^{2}\right)^{\prime}=0$ so that $\phi^{2}$ is constantly equal to $\phi^{2}(0)=0$.

We consider now a classical boundary value problem of the type considered in (3.16). Let $f_{1}, f_{2}: \mathbb{R}^{3} \rightarrow \mathbb{R}$ be continuous and let $a, b, A, B \in \mathbb{R},-\infty<a<b<\infty$. We are looking for solutions $u_{1}, u_{2}:[a, b] \rightarrow \mathbb{R}$ of the system

$$
\begin{aligned}
& u_{1}^{\prime}(x)=f_{1}\left(x, u_{1}(x), u_{2}(x)\right), \\
& u_{2}^{\prime}(x)=f_{2}\left(x, u_{1}(x), u_{2}(x)\right), \\
& u_{1}(a)=A, \\
& u_{1}(b)=B .
\end{aligned}
$$

Theorem 2.1.1 in [3] states the following.

Theorem 3.10. Let $I=] \alpha, \beta],-\infty \leq \alpha<\beta<\infty$, and $\left.I^{0}=\right] \alpha, \beta[$. Assume the following.

i) For every $(x, y) \in I^{0} \times \mathbb{R} z \mapsto f_{1}(x, y, z)$ is an increasing function. Moreover we suppose

$$
\lim _{z \rightarrow \pm \infty} f_{1}(x, y, z)= \pm \infty
$$

uniformly on compact sets in $I^{0} \times \mathbb{R}$.

ii) All the local solutions defined on a subinterval of I of (3.24a) and (3.24b) extend to a solution on the whole interval I.

iii) There exists at most one solution of (3.24), for all $a=a_{0}, b=b_{0} \in I^{0}$ and all $A=A_{0}, B=B_{0} \in \mathbb{R}$. 
Then there exists exactly one solution of (3.24) if $a \in I^{0}$ and $b \in I$.

Previous theorem has an important consequence at the level of existence and uniqueness of solutions to boundary value problems.

Corollary 3.11. Let $F:[a, b] \times \mathbb{R}^{2} \rightarrow \mathbb{R},(x, y, z) \mapsto F(x, y, z)$ be a continuous function. We suppose the following.

i) $(x, y) \mapsto F(x, y, 0)$ has linear growth with respect to $y$.

ii) F fulfills the monotonicity condition (3.18).

iii) F is globally Lipschitz with respect to $z$.

Then there exists exactly one solution to the boundary value problem

$$
\left\{\begin{aligned}
L u(x) & =F\left(x, u, u^{\prime}\right), \\
u(a) & =A \\
u(b) & =B .
\end{aligned}\right.
$$

Proof. Uniqueness follows immediately from Proposition 3.9. To show existence, we make use of Theorem 3.10. Let $\alpha<a$ and $\beta>b$. We extend $F$ continuously on the entire $\mathbb{R}^{3}$ by introducing a new function $\tilde{F}$ in the following way:

$$
\tilde{F}(x, y, z):= \begin{cases}F(a, y, z), & x<a, \\ F(x, y, z), & a \leq x \leq b, \\ F(b, y, z), & x>b .\end{cases}
$$

$F$ fulfills the assumptions of Lipschitz-continuity and monotonicity, and so does $\tilde{F}$. At this point we can show the existence of a unique solution $u_{1}, u_{2}:[a, b] \rightarrow \mathbb{R}$ of the system

$$
\begin{aligned}
& u_{1}^{\prime}(x)=e^{\Sigma(x)} u_{2}(x), \\
& u_{2}^{\prime}(x)=2 \frac{e^{\Sigma(x)}}{\sigma^{2}(x)} \tilde{F}\left(x, u_{1}(x), e^{-\Sigma(x)} u_{2}(x)\right), \\
& u_{1}(a)=A \\
& u_{1}(b)=B .
\end{aligned}
$$

That coincides with (3.24) setting

$$
\begin{aligned}
& f_{1}(x, y, z)=e^{\Sigma(x)} z \\
& f_{2}(x, y, z)=2 \frac{e^{\Sigma(x)}}{\sigma^{2}(x)} \tilde{F}\left(x, y, z e^{-\Sigma(x)}\right) .
\end{aligned}
$$

As the mentioned existence will be a consequence of Theorem 3.10, we check the validity of its assumptions. Clearly, i) is fulfilled. Furthermore, by assumption, $\tilde{F}: \mathbb{R}^{3} \rightarrow \mathbb{R}$ is continuous and has linear growth with respect to $(y, z)$ i.e. the second and third variable. 
Therefore assumption ii) is fulfilled too. Indeed, by Peano's theorem, we can continue (to the left and to the right) locally any solution of (3.27) to a possibly exploding solution. The linear growth condition and Gronwall's lemma imply that no solution explodes. Moreover, Assumption iii) of Theorem 3.10 holds. In fact, since $\tilde{F}$ fulfills the monotonicity condition (3.18) and is globally Lipschitz in $z$, uniqueness follows from Proposition 3.9. Finally, by Lemma 3.8, $u=u_{1}$ is a solution of (3.25).

The proposition below shows existence and uniqueness in the Lipschitz case without the monotonicity condition.

Proposition 3.12. Let $F:[0,1] \times \mathbb{R}^{2} \rightarrow \mathbb{R},(x, y, z) \mapsto F(x, y, z)$ be bounded and globally Lipschitz with respect to $(y, z)$. Then there exists a solution of the boundary value problem

$$
\left\{\begin{aligned}
L u(x) & =F\left(x, u, u^{\prime}\right) \\
u(0) & =A \\
u(1) & =B
\end{aligned}\right.
$$

for any $A, B \in \mathbb{R}$.

Proof. We extend $F$ to $\tilde{F}$ in the way of (3.26) with $a=0$ and $b=1$. Moreover, we define a real function $\Phi: \mathbb{R} \rightarrow \mathbb{R}$ in the following way: for $x_{0}=A$ and $x_{1} \in \mathbb{R}$ we denote the solution of (3.8) by $u^{x_{1}}$. Its existence follows from Proposition 3.6 since $\tilde{F}$ is globally Lipschitz with respect to $(y, z)$. Now we set $\Phi\left(x_{1}\right)=u^{x_{1}}(1)$. Since $\Sigma, F$ and $\sigma$ are continuous, $\Phi$ can be shown to be continuous as well. We leave this to the reader. By (3.9), we get then the following relation:

$$
\Phi\left(x_{1}\right)-x_{0}=\int_{0}^{1} d x e^{-\Sigma(x)}\left(2 \int_{0}^{x} \frac{e^{\Sigma(y)}}{\sigma^{2}(y)} F\left(y, u^{x_{1}}(y),\left(u^{x_{1}}\right)^{\prime}(y)\right) d y+x_{1}\right) .
$$

Since $F$ is bounded,

$$
\lim _{x_{1} \rightarrow \infty} \Phi\left(x_{1}\right)=\infty=-\lim _{x_{1} \rightarrow-\infty} \Phi\left(x_{1}\right) .
$$

Consequently, by mean value theorem, for each $B \in \mathbb{R}$, there is an $x_{1}$ so that $\Phi\left(x_{1}\right)=$ $B$.

Remark 3.13. If $F$ is not bounded, one cannot ensure existence in general. To give an example, we set $L=\frac{d^{2}}{d x^{2}}$ and $F(x, y, z)=-\pi^{2} y$. Then the corresponding boundary value problem

$$
\left\{\begin{aligned}
u^{\prime \prime} & =-\pi^{2} u \\
u(0) & =0 \\
u(1) & =1
\end{aligned}\right.
$$

has no solution. 
Proposition 3.14. Let $a=0, b=1$, and $F:[0,1] \times \mathbb{R} \times \mathbb{R} \rightarrow \mathbb{R},(x, y, z) \mapsto F(x, y, z)$ be globally Lipschitz with respect to $(y, z)$ and Lipschitz-constant $k$, fulfilling

$$
k<\left(\sup _{x \in[0,1]} \int_{0}^{1} d y\left(|K(x, y)|+\left|\partial_{x} K(x, y)\right|\right)\right)^{-1}
$$

where $K$ was defined in (3.3c). Then, (3.14) has a unique solution for any $A, B \in \mathbb{R}$.

Proof. We consider the map $T: C^{1}([0,1]) \rightarrow C^{1}([0,1])$ defined by

$$
T h(x)=f(x)+\int_{0}^{1} K(x, y) F\left(y, h(y), h^{\prime}(y)\right) d y,
$$

with $f$ is given by (3.3b). Taking into account Definition 3.7 and Proposition 3.2, (3.14) is well-posed if and only if $T$ has a fixed point. We show the latter assertion. $C^{1}([0,1])$ is a Banach space equipped with the norm

$$
\|h\|=\sup _{x \in[0,1]}\left(|h(x)|+\left|h^{\prime}(x)\right|\right) .
$$

To show that $T$ admits a unique fixed point, we will show that $T$ is a contraction with respect to $\|\cdot\|$. Let $u, v \in C^{1}([0,1])$. We get

$$
\begin{aligned}
|(T u-T v)(x)| & =\left|\int_{0}^{1} K(x, y)\left(F\left(y, u(y), u^{\prime}(y)\right)-F\left(y, v(y), v^{\prime}(y)\right)\right) d y\right| \\
& \leq \int_{0}^{1} d y|K(x, y)| k\left(|u(y)-v(y)|+\left|u^{\prime}(y)-v^{\prime}(y)\right|\right) \\
& \leq \int_{0}^{1} d y|K(x, y)| k\|u-v\|
\end{aligned}
$$

and

$$
\begin{aligned}
\left|(T u-T v)^{\prime}(x)\right| & =\left|\int_{0}^{1} \partial_{x} K(x, y)\left(F\left(y, u(y), u^{\prime}(y)\right)-F\left(y, v(y), v^{\prime}(y)\right)\right) d y\right| \\
& \leq\left|\int_{0}^{1} d y \partial_{x} K(x, y) k\left(|u(y)-v(y)|+\left|u^{\prime}(y)-v^{\prime}(y)\right|\right) d y\right| \\
& \leq \int_{0}^{1} d y\left|\partial_{x} K(x, y)\right| k\|u-v\| .
\end{aligned}
$$

Summing up (3.31) and (3.32) and taking the supremum over $x$ gives

$$
\|T u-T v\| \leq \sup _{x \in[0,1]} \int_{0}^{1} d y\left(|K(x, y)|+\left|\partial_{x} K(x, y)\right|\right) k\|u-y\| .
$$

It follows that $T$ is a contraction if $k$ fulfills (3.30). 


\section{Exit time of the solution to the forward martingale problem}

We are interested in the nature of the first exit time $\tau$ from the interval $[0,1]$ of a solution $X=X^{x}$ to the martingale problem with respect to $L$ and initial condition $x \in[0,1]$. So we define $\tau$ as

$$
\tau:=\left\{\begin{array}{lc}
\inf \left\{t \geq 0 \mid X_{t} \notin[0,1]\right\}, & \text { if }\left\{t \geq 0 \mid X_{t} \notin[0,1]\right\} \neq \emptyset \\
\infty, & \text { otherwise. }
\end{array}\right.
$$

Proposition 4.1. $\tau$ has finite expectation. In particular $\tau$ is finite almost surely.

Proof. We consider $\Gamma:[0,1] \rightarrow \mathbb{R}$ as the unique solution of

$$
\begin{aligned}
L \Gamma & =-1 \\
\Gamma(0) & =\Gamma(1)=0,
\end{aligned}
$$

and an extension $\tilde{\Gamma} \in \mathcal{D}_{L}$ as regarded in Definition 3.1. Since $X$ is a solution to the martingale problem with respect to $L$ and initial condition $x$, the process

$$
N_{t}=\tilde{\Gamma}\left(X_{t}\right)-\tilde{\Gamma}(x)-\int_{0}^{t} L \tilde{\Gamma}\left(X_{r}\right) d r
$$

is a local martingale. By Proposition 2.14 we have $N_{t}=\int_{0}^{t} \tilde{\Gamma}^{\prime}\left(X_{s}\right) d M_{s}^{X}$, which, by Remark 2.13 i), implies that

$$
[N]_{t}=\int_{0}^{t} \sigma^{2}\left(X_{s}\right) \tilde{\Gamma}^{\prime}\left(X_{s}\right)^{2} d s
$$

Now, let $\left(\tau_{n}\right)$ be the family of stopping times defined as

$$
\tau_{n}:=\inf \left\{t \geq 0 \mid \int_{0}^{t} \sigma^{2}\left(X_{s}\right) \tilde{\Gamma}^{\prime}\left(X_{s}\right)^{2} d s \geq n\right\}
$$

with the assumption that $\inf (\emptyset)=\infty$.

The stopped processes $N^{\tau_{n}}$ are clearly square integrable martingales. By Doob's stopping theorem for martingales, the processes $\left(N_{t \wedge \tau}^{\tau_{n}}\right)_{t \geq 0}$ are again martingales. Consequently,

$$
E\left(\tilde{\Gamma}\left(X_{\tau_{n} \wedge t \wedge \tau}\right)-\tilde{\Gamma}(x)-\int_{0}^{\tau_{n} \wedge t \wedge \tau}(L \tilde{\Gamma})\left(X_{r}\right) d r\right)=0 .
$$

Since $L \tilde{\Gamma}$ restricted to $[0,1]$ equals -1 , the previous expression gives

$$
E\left(\tilde{\Gamma}\left(X_{\tau_{n} \wedge t \wedge \tau}\right)-\tilde{\Gamma}(x)\right)+E\left(\tau_{n} \wedge t \wedge \tau\right)=0
$$

Now we take the limit $n \rightarrow \infty$, and we can use the theorems of monotone and dominated convergence, since

$$
\left|\tilde{\Gamma}\left(X_{\tau_{n} \wedge t \wedge \tau}\right)\right| \leq \sup _{x \in[0,1]}|\Gamma(x)| .
$$


This gives, for every $x \in[0,1]$,

$$
E\left(\Gamma\left(X_{t \wedge \tau}\right)\right)-\Gamma(x)+E(t \wedge \tau)=0 .
$$

Finally, letting $t \rightarrow \infty$, we get

$$
E(\tau)=\Gamma(x)-E\left(\Gamma\left(X_{\tau}\right)\right)=\Gamma(x),
$$

by the same arguments as above taking $n \rightarrow \infty$.

As byproduct of the proof of Proposition 4.1 we get the following.

Proposition 4.2. The expectation of the exit time $\tau$ is exactly $\Gamma(x)$, where $\Gamma$ is the unique solution of

$$
\begin{aligned}
L \Gamma & =-1 \\
\Gamma(0) & =\Gamma(1)=0 .
\end{aligned}
$$

\section{Martingale driven BSDEs with random terminal time}

\subsection{Notion of solution}

The present section does not aim at the greatest generality, which could be the object of future research. We consider the case of one-dimensional BSDEs driven by square integrable martingales with continuous predictable bracket.

Backward SDEs driven by martingales were investigated by several authors, see e. g. [7], [9], see also [12], [8] and [10] for recent developments. We are interested in such a BSDE with terminal condition at random time. This is motivated by the fact that the forward SDE (martingale problem) only admits weak solutions, therefore the reference filtration will only be the canonical one related to the solution and not the one associated with the underlying Brownian motion. We consider the following data.

i) An a.s. finite stopping time $\tau$.

ii) An $\mathcal{F}$-local martingale $\left(M_{t}\right)_{t>0}$ with an $\mathcal{F}$-predictable continuous quadratic variation process $\langle M\rangle$. We suppose moreover that $M^{\tau}$ is an $\mathcal{F}$-square integrable martingale, and we suppose the existence of a deterministic increasing function $\rho: \mathbb{R}_{+} \rightarrow \mathbb{R}_{+}$with $\rho(0)=0$ and

$$
\left\langle M^{\tau}\right\rangle_{t} \leq \rho(t), \forall t \geq 0
$$

iii) A terminal condition $\xi \in L^{2}\left(\Omega, \mathcal{F}_{\tau}, P ; \mathbb{R}\right)$.

iv) A coefficient $f: \Omega \times[0, T] \times \mathbb{R}^{2} \rightarrow \mathbb{R}$, such that the process $f(\cdot, t, y, z), t \geq 0$, is predictable for every $y, z$.

Definition 5.1. Let $(Y, Z, O)$ be a triple of processes with the following properties. 
i) $Y$ is càdlàg $\mathcal{F}$-adapted.

ii) $Z$ is $\mathcal{F}$-predictable such that $E\left(\int_{0}^{\tau} Z_{s}^{2} d\langle M\rangle_{s}\right)<\infty$.

iii) $O$ is a square integrable martingale such that $O_{0}=0$ and $E\left(O_{\tau}^{2}\right)<\infty$. Furthermore, $O$ is strongly orthogonal to $M$, i. e. $\langle M, O\rangle=0$.

iv) $Z_{t}=0$ if $t>\tau$ and $O_{t}=O_{\tau}$ for $t \geq \tau$.

Such a triplet $(Y, Z, O)$ is called solution of the $\operatorname{BSDE}(f, \tau, \xi)$ if it fulfills

$$
Y_{t}=\xi-\int_{t}^{\infty} \mathbb{1}_{\{\tau \geq s\}} Z_{s} d M_{s}+\int_{t}^{\infty} \mathbb{1}_{\{\tau \geq s\}} f\left(\omega, s, Y_{s}, Z_{s}\right) d\langle M\rangle_{s}-\left(O_{\tau}-O_{t \wedge \tau}\right) .
$$

\section{Remark 5.2.}

i) If $t \geq \tau$ in (5.1) we get $Y_{t}=\xi=Y_{\tau}$, so in particular $Y_{t}=Y_{\tau}, t \geq \tau$.

ii) Indeed we will always suppose that $M=M^{\tau}$ so that (5.1) can be rewritten as

$$
Y_{t}=\xi-\int_{t}^{\infty} Z_{s} d M_{s}+\int_{t}^{\infty} f\left(\omega, s, Y_{s}, Z_{s}\right) d\langle M\rangle_{s}-\left(O_{\tau}-O_{t \wedge \tau}\right)
$$

When $M$ is a Brownian motion, this was treated in [11] from which we inherit and adopt very close notations.

\subsection{Uniqueness of Solutions}

Theorem 5.3. Let $a, b, \kappa \in \mathbb{R}$ and set $\gamma=b^{2}-2 a$. We suppose the following.

i) $\left(f\left(\omega, s, y_{1}, z\right)-f\left(\omega, s, y_{2}, z\right)\right)\left(y_{1}-y_{2}\right) \leq-a\left|y_{1}-y_{2}\right|^{2}$, for every $\omega \in \Omega, s \in$ $[0, T], y_{1}, y_{2}, z \in \mathbb{R}$.

ii) $\left|f\left(\omega, s, y, z_{1}\right)-f\left(\omega, s, y, z_{2}\right)\right| \leq b\left|z_{1}-z_{2}\right|$, for every $\omega \in \Omega, s \in[0, T], y \in \mathbb{R}$.

iii) $|f(\omega, s, y, z)-f(\omega, s, 0,0)| \leq \kappa\left(|y|+\kappa^{\prime}\right)+b|z|$, where $\kappa^{\prime} \in\{1,0\}$.

iv) $E\left(\int_{0}^{\tau} e^{\theta\langle M\rangle_{t}}\left(f(t, 0,0)^{2}+\kappa^{\prime}\right) d\langle M\rangle_{t}\right)<\infty$ for every $\theta<\gamma$.

Let $\xi \in L^{2}\left(\Omega, \mathcal{F}_{\tau}\right)$. Then the BSDE $(f, \tau, \xi)$ admits at most one solution $(Y, Z, O)$, such that

$$
E\left(Y_{0}^{2}+\int_{0}^{\tau} e^{\gamma\langle M\rangle_{t}}\left(Y_{t}^{2}+Z_{t}^{2}\right) d\langle M\rangle_{t}+e^{\gamma\langle M\rangle_{t}} d\langle O\rangle_{t}\right)<\infty
$$

\section{Remark 5.4.}

1. In the proof of Theorem 5.3 in appendix B, we omit the dependence of $f$ on $\omega$ in order to simplify the notations.

2. If we suppose $\mathcal{F}_{0}$ to be the trivial $\sigma$-field, then $Y_{0}^{2}$ can be deleted in (5.3). 
In the proof of Theorem 5.3 in appendix $\mathrm{B}$, we use the following technical lemma, which is the generalization of Proposition 4.3 in [11].

Lemma 5.5. Suppose the validity of hypotheses i), ii) and iii) of Theorem 5.3, and let $(Y, Z, O)$ be a solution of $B S D E(f, \tau, \xi)$ such that for some $\theta$,

$$
E\left(Y_{0}^{2}+\int_{0}^{\tau} e^{\theta\langle M\rangle_{s}}\left(\left|Y_{s}\right|^{2}+\left|Z_{s}\right|^{2}+f^{2}(s, 0,0)+\kappa^{\prime}\right) d\langle M\rangle_{s}+\int_{0}^{\tau} e^{\theta\langle M\rangle_{s}} d\langle O\rangle_{s}\right)<\infty .
$$

Then

$$
E\left(\sup _{s \leq \tau} e^{\theta\langle M\rangle_{s}}\left|Y_{s}\right|^{2}\right)<\infty
$$

and

$$
N_{t}=\int_{0}^{t \wedge \tau} e^{\theta\langle M\rangle_{s}} Y_{s-}\left(Z_{s} d M_{s}+d O_{s}\right)=\int_{0}^{t} e^{\theta\langle M\rangle_{s} Y_{s-}}\left(Z_{s} d M_{s}+d O_{s}\right)
$$

is a uniformly integrable martingale.

We prove this lemma in appendix A.

Remark 5.6. Adapting the results of [11] Proposition 3.3, it is possible to state and prove also an existence theorem. We have decided not to do it for two reasons.

1. The techniques can be adapted from the proof of Proposition 3.3 by the same techniques as in the proof of Theorem 5.3.

2. For our applications to the probabilistic representation of semilinear PDEs, we already provide an existence theorem through the resolution of the PDE.

\section{Solutions for BSDEs via solutions of elliptic PDEs}

In this final section we will make the assumptions of Section 2.1 which guarantee existence and uniqueness in law of the martingale problem with respect to $L$. In particular we will suppose that $\sigma>0, \Sigma$ as defined in (2.5) exists and we assume the validity of (2.14) for the function $v$ defined in (2.13).

Let $x_{0} \in \mathbb{R}$ and let $X$ solve a martingale problem $\operatorname{MP}\left(\sigma, \beta ; x_{0}\right)(2.2)$. We are interested in a BSDE with terminal condition at the random time $\tau$, which is the exit time of $X$ from interval $[0,1]$. In this section $\mathcal{F}$ is the canonical filtration $\mathcal{F}^{X}$ of $X$. Since $X$ solves the martingale problem, by Remark 2.13, $X$ is an $\mathcal{F}^{X}$-Dirichlet process. From now on its $\mathcal{F}^{X}$-local martingale component $M^{X}$ will also be denoted by $M$, in agreement with Section 5 .

Let $F: \mathbb{R}^{3} \rightarrow \mathbb{R}$ be a continuous function. We set

$$
f(\omega, t, y, z)=-\frac{F\left(X_{t}(\omega), y, z\right)}{\sigma^{2}\left(X_{t}(\omega)\right)}, \quad t \geq 0, y, z \in \mathbb{R}
$$


and we define

$$
\tau=\inf \left\{t \geq 0 \mid X_{t} \notin I\right\}
$$

Let $u_{0}, u_{1} \in \mathbb{R}$ and set $\xi=\mathbb{1}_{\left\{X_{\tau}=0\right\}} u_{0}+\mathbb{1}_{\left\{X_{\tau}=1\right\}} u_{1}$. So

$$
\xi=u\left(X_{\tau}\right),
$$

for a function $u:[0,1] \rightarrow \mathbb{R}$ such that $u(0)=u_{0}, u(1)=u_{1}$.

Our method allows to construct solutions of the $\operatorname{BSDE}(f, \xi, \tau)$ even in cases that $f$ does not necessarily fulfill Lipschitz or monotonicity assumptions.

We need to check that we are in the framework of the hypotheses at the beginning of Section 5.1.

- i) is verified because of Proposition 4.1.

- ii) holds because

$$
\left\langle M^{\tau}\right\rangle_{t}=\int_{0}^{t \wedge \tau} \sigma^{2}\left(X_{s}\right) d s \leq \rho(t),
$$

where $\rho(t)=t \sup _{x \in[0,1]} \sigma^{2}(x)$.

- iii) is fulfilled since $\xi$ is a bounded random variable, of course $\mathcal{F}_{\tau}$-measurable.

- iv) is verified by construction, and because $X$ is a continuous adapted process.

The aim of this section is to show that the $C^{1}$-type solutions of elliptic PDEs in the sense of Definition 3.7 produce solutions to a BSDE of the type defined in Definition 5.1.

\section{Remark 6.1.}

1. $\mathcal{F}^{X}$ is generally not a Brownian filtration, so that the theory of [11] for existence and uniqueness of BSDEs with random terminal time cannot directly be applied.

2. Even for a simple equation of the type

$$
d X_{t}=\sigma_{0}\left(X_{t}\right) d W_{t},
$$

where $\sigma_{0}$ is only a continuous bounded non-degenerate function, $\mathcal{F}^{X}$ is not necessarily equal to $\mathcal{F}^{W}$ even though $W$ is an $\mathcal{F}^{X}$-Brownian motion.

3. In general, the solution of a semilinear differential equation of the type (3.14) can be associated with the solution of a BSDE driven by the martingale $M^{X}$ which is the martingale component of the $\mathcal{F}^{X}$-Dirichlet process $X$.

4. In Section 5 we have investigated BSDEs driven by (even not continuous) martingales, which are of independent interest. 
Theorem 6.2. Let $I=[0,1]$ and $u: I \rightarrow \mathbb{R}$ be a $C^{1}$-solution of

$$
\left\{\begin{aligned}
L u(x) & =F\left(x, u(x), u^{\prime}(x)\right) \\
u(0) & =u_{0} \\
u(1) & =u_{1} .
\end{aligned}\right.
$$

Let $\left(X_{t}\right)=X^{x_{0}}$ be a solution of $\operatorname{MP}\left(\sigma, \beta ; x_{0}\right)$ on some probability space $(\Omega, \mathcal{G}, P)$. We set, for $t \in[0, T]$,

$$
\begin{aligned}
Y_{t} & =u\left(X_{t}^{\tau}\right) \\
Z_{t} & =u^{\prime}\left(X_{t}\right) \mathbb{1}_{[0, \tau]}(t) \\
O_{t} & =0 .
\end{aligned}
$$

Then $(Y, Z, O)$ is a solution on $(\Omega, \mathcal{G}, P)$ to the $B S D E(f, \xi, \tau)$, where $f, \tau, \xi$ were defined in (6.1), (6.2), (6.3).

Proof. We recall that, by Proposition 4.1, $\tau<\infty$ almost surely.

By Definitions 3.7 and 3.1, there exists $\tilde{u} \in \mathcal{D}_{L}$ which extends $u$ to the real line and $L \tilde{u}=\tilde{\ell}$ and $\tilde{\ell}: \mathbb{R} \rightarrow \mathbb{R}$ is a continuous function extending $\ell(x)=F\left(x, u(x), u^{\prime}(x)\right)$. By the definition of the martingale problem,

$$
M_{t}^{\tilde{u}}:=\tilde{u}\left(X_{t}\right)-\tilde{u}\left(X_{0}\right)-\int_{0}^{t} L \tilde{u}\left(X_{s}\right) d s, t \in[0, T],
$$

is an $\mathcal{F}^{X}$-local martingale.

By Remark 2.6 $\tilde{Y}_{t}=\tilde{u}\left(X_{t}\right)$ is an $\left(\mathcal{F}^{X}\right)$-Dirichlet process with martingale component $\int_{0}^{t} \tilde{u}^{\prime}\left(X_{s}\right) d M_{s}^{X}$. On the other hand, by (6.5), $\tilde{Y}$ is an $\left(\mathcal{F}^{X}\right)$-semimartingale with martingale component $M^{\tilde{u}}$. By uniqueness of decomposition of Dirichlet processes

$$
M_{t}^{\tilde{u}}=\int_{0}^{t} \tilde{u}^{\prime}\left(X_{s}\right) d M_{s}^{X}
$$

We set now

$$
\begin{aligned}
Y_{t} & =\tilde{u}\left(X_{t \wedge \tau}\right) \\
Z_{t} & =\tilde{u}^{\prime}\left(X_{t}\right) \mathbb{1}_{[0, \tau]}(t) .
\end{aligned}
$$

(6.5) gives

$$
\tilde{u}\left(X_{t}\right)-\tilde{u}\left(X_{0}\right)=\int_{0}^{t} L \tilde{u}\left(X_{s}\right) d s+\int_{0}^{t} \tilde{u}^{\prime}\left(X_{s}\right) d M_{s}^{X} .
$$

Stopping previous identity at time $\tau$ implies for every $T>0$ that

$$
Y_{T \wedge \tau}-Y_{t \wedge \tau}=\int_{t \wedge \tau}^{T \wedge \tau} L \tilde{u}\left(X_{s}\right) d s+\int_{t \wedge \tau}^{T \wedge \tau} u^{\prime}\left(X_{s}\right) d M_{s}^{X} .
$$


Letting $T \rightarrow \infty$, since $\tau<\infty$ a. s. gives

$$
Y_{t}=Y_{\tau}-\int_{t \wedge \tau}^{\tau} L \tilde{u}\left(X_{s}\right) d s-\int_{t \wedge \tau}^{\tau} Z_{s} d M_{s}^{X}
$$

So $(Y, Z, O)$ solves BSDE $(f, \tau, \xi)$ with $O \equiv 0$. In particular the conditions of Definition 5.1 are fulfilled. In fact i), iii) and iv) are trivial. ii) holds since $\tilde{u}^{\prime}$ is bounded on $[0,1]$. Moreover (5.1) is fulfilled since $u$ solves (6.4), taking into account (6.1).

Remark 6.3. Since $u$ is bounded, we also have $E\left(\sup _{t \leq \tau} Y_{t}^{2}\right)<\infty$.

By Theorem 6.2, Corollary 3.11 and the Propositions 3.12 and 3.14, we conclude the following.

Corollary 6.4. Let $F:[0,1] \times \mathbb{R}^{2} \rightarrow \mathbb{R}$ be continuous. Suppose that at least one of the following assumptions holds.

a) $(x, y) \mapsto F(x, y, 0)$ has linear growth with respect to $y,(3.18)$ is fulfilled and $F$ is globally Lipschitz in $z$.

b) $(x, y, z) \mapsto F(x, y, z)$ is bounded and globally Lipschitz with respect to $(y, z)$.

c) $(x, y, z) \mapsto F(x, y, z)$ is globally Lipschitz with respect to $(y, z)$ and Lipschitzconstant $k$, fulfilling

$$
k<\left(\sup _{x \in[0,1]} \int_{0}^{1} d y\left(|K(x, y)|+\left|\partial_{x} K(x, y)\right|\right)\right)^{-1},
$$

$K$ being the kernel introduced in (3.3c). Then there is a solution $(Y, Z, O)$ of BSDE $(f, \tau, \xi)$, given by (5.1), where $f, \tau, \xi$ were defined in (6.1), (6.2), (6.3).

Remark 6.5. The solution is provided in the statement of Theorem 6.2.

Corollary 6.6 follows from Corollary 6.4 and Theorem 5.3.

Corollary 6.6. Let $F:[0,1] \times \mathbb{R}^{2} \rightarrow \mathbb{R}$ with the following assumptions.

i) $(x, y) \mapsto F(x, y, 0)$ has linear growth in $y$,

ii) $\left(F\left(x, y_{1}, z\right)-F\left(x, y_{2}, z\right)\right)\left(y_{1}-y_{2}\right) \geq a\left(y_{1}-y_{2}\right)^{2}$ for some $a$,

iii) $F$ is globally Lipschitz in $z$ with constant $b$.

iv) $\gamma=b^{2}-2 a \leq 0$.

Then the solution $(Y, Z, O)$ provided by Corollary 6.4 is unique in the class of

$$
E\left(\int_{0}^{\tau} e^{\gamma\langle M\rangle_{s}} Y_{s}^{2} d\langle M\rangle_{s}+\int_{0}^{\tau} e^{\gamma\langle M\rangle_{s}} Z_{s}^{2} d\langle M\rangle_{s}+\int_{0}^{\tau} e^{\gamma\langle M\rangle_{s}} d\langle O\rangle_{s}\right)<\infty .
$$




\section{Remark 6.7.}

a) Condition iv) implies that $a>0$. In particular $F$ is increasing in $y$.

b) The validity of hypotheses i), ii), iii) imply Hypothesis a) in Corollary 6.4.

c) The solution provided by Corollary 6.4 fulfills (6.6) since $\gamma \leq 0, u, u^{\prime}$ are bounded and $O \equiv 0$, taking into account that $E(\tau)<\infty$, by Proposition 4.1.

Remark 6.8. We discuss an example related to the case $\gamma$ strictly positive, i.e. when Assumption iv) of Corollary 6.6 is not fulfilled. Consider $F(x, y, z)=-\pi^{2} y$.

1. The PDE

$$
\left\{\begin{array}{l}
u^{\prime \prime}(x)=F\left(x, u(x), u^{\prime}(x)\right) \\
u(0)=u(1)=0,
\end{array}\right.
$$

is not well-posed, since $u(x)=\eta \sin (\pi x), \eta \in \mathbb{R}$, provide a class of solutions of (6.7), and so Theorem 6.2 provides a family of solutions of $\operatorname{BSDE}(f, \tau, \xi), \xi \equiv 0, f, \tau, \xi$ being defined in (6.1), (6.2), (6.3), when $X_{t}=\frac{1}{2}+W_{t}$ and $W$ is a standard Brownian motion. In particular $X$ solves $\operatorname{MP}\left(\sigma, \beta ; x_{0}\right)$ in the sense of Definition 2.9 with $x_{0}=\frac{1}{2}, \sigma=1, \beta=0$. We remark that $a=-\pi^{2}, b=0$, so $\gamma=b^{2}-2 a=2 \pi^{2}>0$.

2. In that case, since the mentioned Assumption iv) is not fulfilled, then of course Corollary 6.6 cannot be applied. Indeed, if $\eta \neq 0$, the solutions, provided explicitly above are not in the class of solutions fulfilling (6.6), as we show below.

Let $\tau$ be the exit time of Brownian motion $X$ starting from $x_{0}=\frac{1}{2}$ from interval $[0,1]$. By Proposition C. 1 in appendix C we have $E(\exp (\gamma \tau))=\frac{1}{\cos \left(\sqrt{\frac{\gamma}{2}}\right)}$ if $0 \leq \gamma<$ $\frac{\pi^{2}}{2}$, and $E(\exp (\gamma \tau))=\infty$ whenever $\gamma \geq \frac{\pi^{2}}{2}$. Consequently if $\gamma=2 \pi^{2}$ as before, then $E(\exp (\gamma \tau))=\infty$.

We have $\langle M\rangle_{t} \equiv t, O \equiv 0$. So, (6.6) gives

$$
\begin{aligned}
E\left(\int_{0}^{\tau} d s e^{\gamma s} \eta^{2}\left(\sin ^{2}\left(\pi X_{s}\right)+\pi^{2} \cos ^{2}\left(\pi X_{s}\right)\right)\right) & \\
\geq E\left(\int_{0}^{\tau} e^{\gamma s} \eta^{2} d s\right) & =\frac{\eta^{2}}{\gamma} E\left(e^{\gamma \tau}\right)=\infty,
\end{aligned}
$$

since $\eta \neq 0$. In conclusion, if $\gamma>0$, the solutions provided by Corollary 6.4 may fulfill or not (6.6).

3. On the other hand the solutions above fulfill the version of (6.6) with $\gamma=0$, i.e.

$$
E\left(\int_{0}^{\tau}\left(Y_{t}^{2}+Z_{t}^{2}\right) d\langle M\rangle_{t}+d\langle O\rangle_{t}\right)<\infty
$$

since $\tau$ has finite expectation and $u, u^{\prime}$ are bounded. In particular the class of solutions fulfilling only (6.8) is not, in general, a good class for uniqueness. 


\section{Appendix}

\section{A. Proof of Lemma 5.5}

Since $Y$ solves the BSDE, by integration by parts we get

$$
\begin{aligned}
e^{\frac{\theta}{2}\langle M\rangle_{t \wedge \tau} Y_{t \wedge \tau}=Y_{0}} & +\int_{0}^{t \wedge \tau} e^{\frac{\theta}{2}\langle M\rangle_{s}}\left(Z_{s} d M_{s}+d O_{s}\right) \\
& -\int_{0}^{t \wedge \tau} e^{\frac{\theta}{2}\langle M\rangle_{s}} f\left(s, Y_{s}, Z_{s}\right) d\langle M\rangle_{s}+\frac{\theta}{2} \int_{0}^{t \wedge \tau} e^{\frac{\theta}{2}\langle M\rangle_{s}} Y_{s} d\langle M\rangle_{s} .
\end{aligned}
$$

By Assumption ii) of Section 5.1, $\langle M\rangle$ is continuous. Consequently,

$$
\left[e^{\frac{\theta}{2}\langle M\rangle} Y\right]_{t \wedge \tau}=\left[N^{\theta}\right]_{t \wedge \tau}
$$

where

$$
N_{t}^{\theta}:=\int_{0}^{t} e^{\frac{\theta}{2}\langle M\rangle_{s}}\left(Z_{s} d M_{s}+d O_{s}\right) .
$$

Remark A.1. From (5.4) it follows that

$$
E\left(\int_{0}^{\tau} e^{\theta\langle M\rangle_{s}}\left(Z_{s}^{2} d\langle M\rangle_{s}+d\langle O\rangle_{s}\right)\right)<\infty
$$

Consequently, $N^{\theta}$ is a square integrable martingale. So, by the proof of Proposition 4.50 in [18], there is a uniformly integrable martingale $\mathcal{M}^{\theta}$, so that

$$
\left[N^{\theta}\right]=\left\langle N^{\theta}\right\rangle+\mathcal{M}^{\theta}
$$

We continue with the proof of Lemma 5.5 by using Itô's formula and (A.1) getting

$$
\begin{aligned}
e^{\theta\langle M\rangle_{t \wedge \tau}} Y_{t \wedge \tau}^{2}-Y_{0}^{2}= & \left(e^{\frac{\theta}{2}\langle M\rangle_{t \wedge \tau}} Y_{t \wedge \tau}\right)^{2}-Y_{0}^{2} \\
= & 2 \int_{0}^{t \wedge \tau} e^{\frac{\theta}{2}\langle M\rangle_{s}} Y_{s-} d\left(e^{\frac{\theta}{2}\langle M\rangle_{s}} Y_{s}\right)+\left[e^{\frac{\theta}{2}\langle M\rangle} Y\right]_{t \wedge \tau} \\
= & 2 \int_{0}^{t \wedge \tau} e^{\theta\langle M\rangle_{s}} Y_{s-}\left(Z_{s} d M_{s}+d O_{s}\right) \\
& -2 \int_{0}^{t \wedge \tau} e^{\theta\langle M\rangle_{s}} Y_{s} f\left(s, Y_{s}, Z_{s}\right) d\langle M\rangle_{s} \\
& +2 \frac{\theta}{2} \int_{0}^{t \wedge \tau} e^{\theta\langle M\rangle_{s}} Y_{s}^{2} d\langle M\rangle_{s}+\left[N^{\theta}\right]_{t \wedge \tau},
\end{aligned}
$$

where in the latter equality we have taken into account (A.2). Since $\langle M\rangle$ is continuous we have been allowed to replace $Y_{s-}$ with $Y_{s}$ in the two lines above. By use of CauchySchwarz, the inequality $2 \alpha \beta \leq \alpha^{2}+\beta^{2}$ and assumption iii) of Theorem 5.3, there is a 
constant $c$, depending on $\kappa, b$ and $\theta$, such that

$$
\begin{aligned}
e^{\theta\langle M\rangle_{t \wedge \tau}} Y_{t \wedge \tau}^{2}- & Y_{0}^{2} \leq c \int_{0}^{t \wedge \tau} e^{\theta\langle M\rangle_{s}}\left(Y_{s}^{2}+Z_{s}^{2}\right. \\
& \left.+f^{2}(s, 0,0)+\kappa^{\prime}\right) d\langle M\rangle_{s}+2 \int_{0}^{t \wedge \tau} e^{\frac{\theta}{2}\langle M\rangle_{s} Y_{s-}} d N_{s}^{\theta}+\left[N^{\theta}\right]_{t \wedge \tau} .
\end{aligned}
$$

Now we continue with a localization of (A.5). For that we define for each $n \in \mathbb{N}$ a stopping time $\tau(n)$ by

$$
\tau(n):=\inf \left\{t \mid Y_{t} \geq n\right\} \wedge n .
$$

Replacing $t$ with $t \wedge \tau(n)$ in (A.5) gives

$$
\begin{aligned}
& e^{\theta\langle M\rangle_{t \wedge \tau(n) \wedge \tau}} Y_{t \wedge \tau(n) \wedge \tau}^{2}-Y_{0}^{2} \leq c \int_{0}^{t \wedge \tau(n) \wedge \tau} e^{\theta\langle M\rangle_{s}}\left(Y_{s}^{2}+Z_{s}^{2}\right. \\
& \left.\quad+f^{2}(s, 0,0)+\kappa^{\prime}\right) d\langle M\rangle_{s}+2 \int_{0}^{t \wedge \tau(n) \wedge \tau} e^{\frac{\theta}{2}\langle M\rangle_{s}} Y_{s-} d N_{s}^{\theta}+\left[N^{\theta}\right]_{t \wedge \tau(n) \wedge \tau} .
\end{aligned}
$$

We take the supremum over $t$ on the left-hand side and afterwards the expectation. Recalling that

$$
N_{t}=\int_{0}^{t} e^{\frac{\theta}{2}\langle M\rangle_{s}} Y_{s-} d N_{s}^{\theta}
$$

this yields

$$
\begin{aligned}
E\left(\sup _{t \leq \tau(n) \wedge \tau}\left(e^{\theta\langle M\rangle_{t \wedge \tau(n) \wedge \tau}} Y_{t \wedge \tau(n) \wedge \tau}^{2}\right)\right) & \\
& \leq E\left(Y_{0}^{2}\right)+c E(\mathcal{D})+2 E\left(\sup _{t \geq 0}\left|N_{t}^{\tau(n) \wedge \tau}\right|\right)+E\left(\left[N^{\theta}\right]_{\tau(n) \wedge \tau}\right)
\end{aligned}
$$

where

$$
\mathcal{D}=\int_{0}^{\tau} e^{\theta\langle M\rangle_{s}}\left(Y_{s}^{2}+Z_{s}^{2}+f^{2}(s, 0,0)+\kappa^{\prime}\right) d\langle M\rangle_{s},
$$

which has finite expectation because of (5.4). By Remark A.1,

$$
\begin{aligned}
E\left(\left[N^{\theta}\right]_{\tau(n) \wedge \tau}\right) & =E\left(\left\langle N^{\theta}\right\rangle_{\tau(n) \wedge \tau}\right) \\
& =E\left(\int_{0}^{\tau(n) \wedge \tau} e^{\theta\langle M\rangle_{s}}\left(Z_{s}^{2} d\langle M\rangle_{s}+d\langle O\rangle_{s}\right)\right) .
\end{aligned}
$$


We show now that $N^{\tau(n) \wedge \tau}$ is a square integrable martingale. This happens because by (5.6) we have

$$
\begin{aligned}
E\left(\langle N\rangle_{\tau(n) \wedge \tau}\right) & =E\left(\int_{0}^{\tau(n) \wedge \tau} e^{2 \theta\langle M\rangle_{s}} Y_{s}^{2}\left(Z_{s}^{2} d\langle M\rangle_{s}+d\langle O\rangle_{s}\right)\right) \\
& \leq n^{2} E\left(\int_{0}^{\tau \wedge n} e^{2 \theta\langle M\rangle_{s}}\left(Z_{s}^{2} d\langle M\rangle_{s}+d\langle O\rangle_{s}\right)\right) \\
& \leq n^{2} e^{\theta \rho(n)} E\left(\int_{0}^{\tau} e^{\theta\langle M\rangle_{s}}\left(Z_{s}^{2} d\langle M\rangle_{s}+d\langle O\rangle_{s}\right)\right)<\infty
\end{aligned}
$$

taking into account Assumption ii) at the beginning of Section 5.1. So by Proposition 4.50 of [18], there is a uniformly integrable martingale $\tilde{\mathcal{M}}$ so that

$$
\left[N^{\tau(n) \wedge \tau}\right]=\left\langle N^{\tau(n) \wedge \tau}\right\rangle+\tilde{\mathcal{M}}
$$

Due to the Burkholder-Davis-Gundy (BDG) inequalities (see e.g. [22, Theorem IV.48]), there is a constant $c_{0}$ such that

$$
E\left(\sup _{t \geq 0}\left|N_{t}^{\tau(n) \wedge \tau}\right|\right) \leq c_{0} E\left([N, N]_{\tau(n) \wedge \tau}^{\frac{1}{2}}\right)
$$

We denote by $\mathcal{N}$ the local martingale

$$
\mathcal{N}_{t}=\int_{0}^{t} Z_{s} d M_{s}+O_{t}
$$

By Theorem 29 in Chapter II of [22] the right-hand side of (A.11) equals

$$
\begin{gathered}
c_{0} E\left(\left(\int_{0}^{\tau(n) \wedge \tau} e^{\theta\langle M\rangle_{s}} e^{\theta\langle M\rangle_{s}} Y_{s}^{2} d[\mathcal{N}]_{s}\right)^{\frac{1}{2}}\right) \\
\quad \leq c_{0} E\left(\left(\sup _{t \leq \tau(n) \wedge \tau}\left(e^{\theta\langle M\rangle_{t}} Y_{t}^{2}\right)\right)^{\frac{1}{2}}\left(\int_{0}^{\tau(n) \wedge \tau} e^{\theta\langle M\rangle_{s}} d[\mathcal{N}]_{s}\right)^{\frac{1}{2}}\right) .
\end{gathered}
$$

By $2 \alpha \beta \leq \frac{\alpha^{2}}{c_{3}}+c_{3} \beta^{2}$, for any $c_{3}>0$, the right-hand side of (A.12) is bounded by

$$
\begin{array}{r}
\frac{c_{0}}{2 c_{3}} E\left(\sup _{t \leq \tau(n) \wedge \tau}\left(e^{\theta\langle M\rangle_{t}} Y_{t}^{2}\right)\right)+\frac{c_{0} c_{3}}{2} E\left(\int_{0}^{\tau(n) \wedge \tau} e^{\theta\langle M\rangle_{s}} d[\mathcal{N}]_{s}\right) \\
=\frac{c_{0}}{2 c_{3}} E\left(\sup _{t \leq \tau(n) \wedge \tau}\left(e^{\theta\langle M\rangle_{t}} Y_{t}^{2}\right)\right)+\frac{c_{0} c_{3}}{2} E\left(\left[N^{\theta}\right]_{\tau(n) \wedge \tau}\right),
\end{array}
$$


also using (A.3) and [22], Theorem 29, Chapter II. This gives, by (A.11), (A.10) and (A.12),

$$
\begin{aligned}
E\left(\sup _{t \geq 0}\left|N_{t}^{\tau(n) \wedge \tau}\right|\right) \leq \frac{c_{0}}{2 c_{3}} E & \left(\sup _{t \leq \tau(n) \wedge \tau}\left(e^{\theta\langle M\rangle_{t}} Y_{t}^{2}\right)\right) \\
& +\frac{c_{0} c_{3}}{2} E\left(\int_{0}^{\tau(n) \wedge \tau} e^{\theta\langle M\rangle_{s}}\left(Z_{s}^{2} d\langle M\rangle_{s}+d\langle O\rangle_{s}\right)\right) .
\end{aligned}
$$

Plugging (A.10) and (A.14) in (A.8) gives

$$
\begin{aligned}
E\left(\sup _{t \leq \tau(n) \wedge \tau}\left(e^{\theta\langle M\rangle_{t}} Y_{t}^{2}\right)\right) & \\
& \leq E\left(Y_{0}^{2}\right)+E(\mathcal{D})\left(1+c+c_{0} c_{3}\right)+\frac{c_{0}}{c_{3}} E\left(\sup _{t \leq \tau(n) \wedge \tau}\left(e^{\theta\langle M\rangle_{t}} Y_{t}^{2}\right)\right) .
\end{aligned}
$$

Choosing $c_{3}=2 c_{0}$, we get

$$
E\left(\sup _{t \leq \tau(n) \wedge \tau}\left(e^{\theta\langle M\rangle_{t}} Y_{t}^{2}\right)\right) \leq 2 E\left(Y_{0}^{2}\right)+2 E(\mathcal{D})\left(1+c+2 c_{0}^{2}\right) .
$$

By the monotone convergence theorem, letting $n \rightarrow \infty$, we get

$$
E\left(\sup _{t \leq \tau}\left(e^{\theta\langle M\rangle_{t}} Y_{t}^{2}\right)\right) \leq 2 E\left(Y_{0}^{2}\right)+2 E(\mathcal{D})\left(1+c+2 c_{0}^{2}\right)
$$

which shows (5.5).

We go on with the second part, i. e. the fact that $N$ defined in (5.6) is a uniformly integrable martingale. By BDG and Cauchy-Schwarz inequalities,

$$
\begin{gathered}
E\left(\sup _{t \geq 0}\left|N_{t}\right|\right) \leq c_{0} E\left([N, N]^{\frac{1}{2}}\right) \leq c_{0} E\left(\left(\int_{0} e^{2 \theta\langle M\rangle_{s}} Y_{s-}^{2} d[\mathcal{N}]_{s}\right)^{\frac{1}{2}}\right) \\
\leq c_{0} E\left(\left(\sup _{t \leq \tau} e^{\theta\langle M\rangle_{t}} Y_{t}^{2}\right)^{\frac{1}{2}}\left(\int_{0}^{\tau} e^{\theta\langle M\rangle_{s}} d[\mathcal{N}]_{s}\right)^{\frac{1}{2}}\right) \\
\leq c_{0}\left(E\left(\sup _{t \leq \tau} e^{\theta\langle M\rangle_{t}} Y_{t}^{2}\right)\right)^{\frac{1}{2}}\left(E\left(\int_{0}^{\tau} e^{\theta\langle M\rangle_{s}} d[\mathcal{N}]_{s}\right)\right)^{\frac{1}{2}} \\
=c_{0}\left(E\left(\sup _{t \leq \tau} e^{\theta\langle M\rangle_{t}} Y_{t}^{2}\right)\right)^{\frac{1}{2}}\left(E\left(\left[N^{\theta]}\right]_{\tau}\right)\right)^{\frac{1}{2}} .
\end{gathered}
$$

By Remark A.1

$$
E\left(\left[N^{\theta}\right]_{\tau}\right)=E\left(\left\langle N^{\theta}\right\rangle_{\tau}\right)=E\left(\int_{0}^{\tau} e^{\theta\langle M\rangle_{s}}\left(Z_{s}^{2} d\langle M\rangle_{s}+d\langle O\rangle_{s}\right)\right)<\infty .
$$

This shows that $N$ is a uniformly integrable martingale and finally, Lemma 5.5 is established. 


\section{B. Proof of Theorem 5.3}

We start with some a priori bounds. Let $\theta<\gamma$. By assumptions i) and ii), for any $\varepsilon \geq 0$, using $2 \alpha \beta \leq \frac{\alpha^{2}}{1+\varepsilon}+(1+\varepsilon) \beta^{2}$, we can easily show that

$$
2(y-\bar{y})(f(s, y, z)-f(s, \bar{y}, \bar{z})) \leq-2 a|y-\bar{y}|^{2}+b^{2}(1+\varepsilon)|y-\bar{y}|^{2}+\frac{|z-\bar{z}|^{2}}{1+\varepsilon} .
$$

Let $\left(Y^{i}, Z^{i}, O^{i}\right), i=1,2$ be two solutions fulfilling (5.3) of the statement. By similar arguments as (A.4) and in the lines before, for $Y=Y^{1}-Y^{2}, Z=Z^{1}-Z^{2}, O=O^{1}-O^{2}$ we have

$$
\begin{gathered}
e^{\theta\langle M\rangle_{\tau}} Y_{\tau}^{2}-e^{\theta\langle M\rangle_{t \wedge \tau}} Y_{t \wedge \tau}^{2}=\int_{t \wedge \tau}^{\tau} \theta e^{\theta\langle M\rangle_{s}} Y_{s}^{2} d\langle M\rangle_{s}+2 \int_{t \wedge \tau}^{\tau} e^{\frac{\theta}{2}\langle M\rangle_{s}} Y_{s-} d N_{s}^{\theta} \\
-2 \int_{t \wedge \tau}^{\tau} e^{\theta\langle M\rangle_{s}} Y_{s}\left(f\left(s, Y_{s}^{1}, Z_{s}^{1}\right)-f\left(s, Y_{s}^{2}, Z_{s}^{2}\right)\right) d\langle M\rangle_{s}+\left[N^{\theta}\right]_{\tau}-\left[N^{\theta}\right]_{t \wedge \tau},
\end{gathered}
$$

where $N^{\theta}$ was defined in (A.3). By (B.1) we get

$$
\begin{aligned}
2 \int_{t \wedge \tau}^{\tau} e^{\theta\langle M\rangle_{s}} & Y_{s}\left(f\left(s, Y_{s}^{1}, Z_{s}^{1}\right)-f\left(s, Y_{s}^{2}, Z_{s}^{2}\right)\right) d\langle M\rangle_{s} \\
\leq & \int_{t \wedge \tau}^{\tau} e^{\theta\langle M\rangle_{s}}\left(b^{2}(1+\varepsilon)-2 a\right) Y_{s}^{2} d\langle M\rangle_{s}+\int_{t \wedge \tau}^{\tau} e^{\theta\langle M\rangle_{s}} \frac{\left|Z_{s}\right|^{2}}{1+\varepsilon} d\langle M\rangle_{s} .
\end{aligned}
$$

$\left(Y^{i}, Z^{i}, O^{i}\right), i=1,2$ fulfills (5.4) by (5.3) and Assumption iv) of Theorem 5.3. Consequently $(Y, Z, O)$ also fulfills (5.4). By Remark A.1, since $\theta<\gamma, N^{\theta}$ is a square integrable martingale and

$$
\left[N^{\theta}\right]=\left\langle N^{\theta}\right\rangle+\mathcal{M}^{\theta}
$$

where $\mathcal{M}^{\theta}$ is a uniformly integrable martingale. So

$$
E\left(\left[N^{\theta}\right]_{\tau}-\left[N^{\theta}\right]_{t \wedge \tau}\right)=E\left(\int_{t \wedge \tau}^{\tau} e^{\theta\langle M\rangle_{s}}\left(Z_{s}^{2} d\langle M\rangle_{s}+d\langle O\rangle_{s}\right)\right) .
$$

(B.2), (B.3) and the fact that $Y_{\tau}=0$, gives

$$
\begin{aligned}
& e^{\theta\langle M\rangle_{t \wedge \tau}} Y_{t \wedge \tau}^{2}+\left[N^{\theta}\right]_{\tau}-\left[N^{\theta}\right]_{t \wedge \tau}+2 \int_{t \wedge \tau}^{\tau} e^{\frac{\theta}{2}\langle M\rangle_{s}} Y_{s-} d N_{s}^{\theta} \\
& \leq \int_{t \wedge \tau}^{\tau} e^{\theta\langle M\rangle_{s}}\left(b^{2}(1+\varepsilon)-2 a-\theta\right) Y_{s}^{2} d\langle M\rangle_{s}+\int_{t \wedge \tau}^{\tau} e^{\theta\langle M\rangle_{s}} \frac{Z_{s}^{2}}{1+\varepsilon} d\langle M\rangle_{s} .
\end{aligned}
$$

By Lemma 5.5, since $\theta<\gamma$,

$$
\left(\int_{0}^{t \wedge \tau} e^{\frac{\theta}{2}\langle M\rangle_{s}} Y_{s-} d N_{s}^{\theta}\right)_{t \geq 0}
$$


is a uniformly integrable martingale. So its expectation is zero. By previous considerations, (B.3) and (B.4), we take the expectation in (B.5) to get

$$
\begin{aligned}
E\left(e^{\theta\langle M\rangle_{t \wedge \tau}} Y_{t \wedge \tau}^{2}+\int_{t \wedge \tau}^{\tau} e^{\theta\langle M\rangle_{s}}\right. & \left.\left(\frac{\varepsilon Z_{s}^{2}}{1+\varepsilon} d\langle M\rangle_{s}+d\langle O\rangle_{s}\right)\right) \\
& \leq E\left(\int_{t \wedge \tau}^{\tau} e^{\theta\langle M\rangle_{s}}\left(b^{2}(1+\varepsilon)-2 a-\theta\right) Y_{s}^{2} d\langle M\rangle_{s}\right) .
\end{aligned}
$$

Since $\theta<\gamma=b^{2}-2 a$, we have $b^{2}(1+\varepsilon)-2 a-\theta>0, \forall \varepsilon \geq 0$. We let $\varepsilon \rightarrow 0$ so that (B.6) becomes

$$
\begin{aligned}
& E\left(e^{\theta\langle M\rangle_{t \wedge \tau}} Y_{t \wedge \tau}^{2}+\int_{t \wedge \tau}^{\tau} e^{\theta\langle M\rangle_{s}} d\langle O\rangle_{s}\right) \\
& \leq E\left(\int_{t \wedge \tau}^{\tau} e^{\theta\langle M\rangle_{s}}\left(b^{2}-2 a-\theta\right) Y_{s}^{2} d\langle M\rangle_{s}\right) .
\end{aligned}
$$

Equation (B.7) holds for every $\theta<\gamma$. We let $\theta \rightarrow \gamma-$. By the monotone convergence theorem we get

$$
E\left(e^{\gamma\langle M\rangle_{t \wedge \tau}} Y_{t \wedge \tau}^{2}+\int_{t \wedge \tau}^{\tau} e^{\gamma\langle M\rangle_{s}} d\langle O\rangle_{s}\right) \leq 0
$$

Equation (B.8) finally shows that $Y \equiv 0$ and $\langle O\rangle \equiv 0$. Coming back to (B.6), it easily follows that $Z \equiv 0 d\langle M\rangle$ a.s.

\section{Exponential moments of the first exit time of Brownian motion}

We consider a standard one-dimensional Brownian motion $\left\{W=W_{t}: t \geq 0\right\}, x_{0}, a, b \in \mathbb{R}$ so that $\left.x_{0} \in\right] a, b[$. We consider the exit time

$$
\tau=\min \left\{s: x_{0}+W_{s} \notin\right] a, b[\},
$$

from the interval $] a, b[$. According to [5, p. 212],

$$
E\left(e^{\gamma \tau}\right)=\frac{\cosh \left(\left(b+a-2 x_{0}\right) \sqrt{-\frac{\gamma}{2}}\right)}{\cosh \left((b-a) \sqrt{-\frac{\gamma}{2}}\right)}, \quad \gamma \leq 0 .
$$

We define the function $f$ by

$$
\begin{aligned}
f: \mathbb{C} & \rightarrow \mathbb{C}, \\
z & \mapsto \sum_{n=0}^{\infty}(-1)^{n} \frac{z^{n}}{(2 n) !} .
\end{aligned}
$$


Clearly $f$ is analytical. For $x \in \mathbb{R}$ we can verify by Taylor series expansion that

$$
f(x)= \begin{cases}\cos \sqrt{x}, & x \geq 0, \\ \cosh \sqrt{-x}, & x<0 .\end{cases}
$$

Let $g$ be another analytical function, defined by

$$
\begin{aligned}
g: \mathbb{C} \backslash\left\{\frac{\pi^{2}(2 k+1)^{2}}{2(b-a)^{2}}, k \in \mathbb{N}\right\} & \rightarrow \mathbb{C}, \\
\gamma & \mapsto \frac{f\left(\frac{\left(b+a-2 x_{0}\right)^{2}}{2} \gamma\right)}{f\left(\frac{(b-a)^{2}}{2} \gamma\right)} .
\end{aligned}
$$

In particular, for $\gamma \in \mathbb{R}$ and $\gamma \leq 0$, this gives

$$
g(\gamma)=\frac{\cosh \left(\left(b+a-2 x_{0}\right) \sqrt{-\frac{\gamma}{2}}\right)}{\cosh \left((b-a) \sqrt{-\frac{\gamma}{2}}\right)} .
$$

Proposition C.1. Let $\gamma \geq 0$.

i) $E\left(e^{\gamma \tau}\right)=g(\gamma), \gamma<\frac{\pi^{2}}{2(b-a)^{2}}$

ii) $E\left(e^{\gamma \tau}\right)=\infty, \gamma \geq \frac{\pi^{2}}{2(b-a)^{2}}$.

Proof. ii) follows from i), since $\gamma \mapsto E\left(e^{\gamma \tau}\right)$ is monotone, taking into account the BeppoLevi convergence theorem. By (C.2) and (C.6), i) holds for $\gamma \leq 0$, and it remains to show i) in the case $0<\gamma<\frac{\pi^{2}}{2(b-a)^{2}}$. For $\gamma<0$ and $n \in \mathbb{N}$,

$$
E\left(\tau^{n} e^{\gamma \tau}\right)=\frac{d^{n}}{d \gamma^{n}} g(\gamma)
$$

Again, by the monotone convergence theorem, letting $\gamma \rightarrow 0-$, we get

$$
E\left(\tau^{n}\right)=\left.\frac{d^{n}}{d \gamma^{n}} g(\gamma)\right|_{\gamma=0}
$$

In particular all moments of $\tau$ exist. Now if $0<\gamma<\frac{\pi^{2}}{2(b-a)^{2}}$, then by Fubini we get

$$
\begin{aligned}
E\left(e^{\gamma \tau}\right)=\sum_{n=0}^{\infty} \frac{\gamma^{n}}{n !} E\left(\tau^{n}\right)=\left.\sum_{n=0}^{\infty} \frac{\gamma^{n}}{n !} \frac{d^{n}}{d \gamma^{n}} g(\gamma)\right|_{\gamma=0}= & g(\gamma) \\
& =\frac{\cos \left(\left(b+a-2 x_{0}\right) \sqrt{\frac{\gamma}{2}}\right)}{\cos \left((b-a) \sqrt{\frac{\gamma}{2}}\right)}
\end{aligned}
$$

since $g$ is analytical on its domain. Finally i) follows. 
ACKNOWLEDGEMENTS: The authors are grateful to the Referee for her / his interesting comments and suggestions.

The research was partially supported by the ANR Project MASTERIE 2010 BLAN0121-01. The first named author also benefited partially from the support of the "FMJH Program Gaspard Monge in optimization and operation research" (Project 2014-1607H). The second named author was supported by a "Marietta-Blau-Stipendium" coming from the Austrian federal Ministry of science, research and economy (BMWF).

\section{References}

[1] Bass, R. F. and Chen, Z.-Q. "Brownian motion with singular drift". In: Ann. Probab. 31.2 (2003), pp. 791-817.

[2] Bass, R. F. and Chen, Z.-Q. "Stochastic differential equations for Dirichlet processes". In: Probab. Theory Related Fields 121.3 (2001), pp. 422-446.

[3] Bernfeld, S. R. and Lakshmikantham, V. An introduction to nonlinear boundary value problems. Mathematics in Science and Engineering, Vol. 109. Academic Press, Inc. [A subsidiary of Harcourt Brace Jovanovich, Publishers], New York-London, 1974, pp. xi+386.

[4] Bertoin, J. "Les processus de Dirichlet en tant qu'espace de Banach". In: Stochastics 18.2 (1986), pp. 155-168.

[5] Borodin, A. N. and Salminen, P. Handbook of Brownian motion-facts and formulae. Second. Probability and its Applications. Birkhäuser Verlag, Basel, 2002, pp. xvi+672.

[6] Briand, P., Delyon, B., and Mémin, J. "On the robustness of backward stochastic differential equations". In: Stochastic Processes and their Applications 97.2 (2002), pp. 229-253.

[7] Buckdahn, R. "Backward Stochastic Differential Equations Driven by a Martingale". In: Preprint, Humboldt University, Berlin (1993).

[8] Buckdahn, R. and Engelbert, H.-J. "A backward stochastic differential equation without strong solution". In: Teor. Veroyatn. Primen. 50.2 (2005), pp. 390-396.

[9] Carbone, R., Ferrario, B., and Santacroce, M. "Backward stochastic differential equations driven by càdlàg martingales". In: Teor. Veroyatn. Primen. 52.2 (2007), pp. $375-385$.

[10] Ceci, C., Cretarola, A., and Russo, F. "BSDEs under partial information and financial applications." In: Stochastic processes and applications. 124 (8) (Mar. 2014), 26282653.

[11] Darling, R. W. R. and Pardoux, E. "Backwards SDE with random terminal time and applications to semilinear elliptic PDE". In: Ann. Probab. 25.3 (1997), pp. 11351159. 
[12] El Karoui, N. and Huang, S.-J. "A general result of existence and uniqueness of backward stochastic differential equations". In: Backward stochastic differential equations (Paris, 1995-1996). Vol. 364. Pitman Res. Notes Math. Ser. Harlow: Longman, 1997, pp. 27-36.

[13] Flandoli, F., Issoglio, E., and Russo, F. "Multidimensional stochastic differential equations with distributional drift". In: Transactions of the American Mathematical Society, to appear. (2014). http://hal.inria.fr/hal-00935399.

[14] Flandoli, F., Russo, F., and Wolf, J. "Some SDEs with distributional drift. I. General calculus". In: Osaka J. Math. 40.2 (2003), pp. 493-542.

[15] Flandoli, F., Russo, F., and Wolf, J. "Some SDEs with distributional drift. II. Lyons-Zheng structure, Itô's formula and semimartingale characterization". In: Random Oper. Stochastic Equations 12.2 (2004), pp. 145-184.

[16] Föllmer, H. "Calcul d'Itô sans probabilités". In: Seminar on Probability, XV (Univ. Strasbourg, Strasbourg, 1979/1980) (French). Vol. 850. Lecture Notes in Math. Berlin: Springer, 1981, pp. 143-150.

[17] $\mathrm{Hu}, \mathrm{Y}$. and Shi, Z. "The limits of Sinai's simple random walk in random environment". In: Ann. Probab. 26.4 (1998), pp. 1477-1521.

[18] Jacod, J. and Shiryaev, A. N. Limit theorems for stochastic processes. Second. Vol. 288. Grundlehren der Mathematischen Wissenschaften [Fundamental Principles of Mathematical Sciences]. Springer-Verlag, Berlin, 2003, pp. xx+661.

[19] Karatzas, J. and Ruf, I. "Pathwise solvability of stochastic integral equations with generalized drift and non-smooth dispersion functions". In: preprint (2013). arXiv:1312.7257v1 [math.PR].

[20] Mathieu, P. "Limit theorems for diffusions with a random potential". In: Stochastic Process. Appl. 60.1 (1995), pp. 103-111.

[21] Mathieu, P. "Zero white noise limit through Dirichlet forms, with application to diffusions in a random medium". In: Probab. Theory Related Fields 99.4 (1994), pp. 549-580.

[22] Protter, P. E. Stochastic integration and differential equations. Second. Vol. 21. Applications of Mathematics (New York). Stochastic Modelling and Applied Probability. Springer-Verlag, Berlin, 2004, pp. xiv+415.

[23] Russo, F. and Trutnau, G. "Some parabolic PDEs whose drift is an irregular random noise in space". In: Ann. Probab. 35.6 (2007), pp. 2213-2262.

[24] Russo, F. and Vallois, P. "Elements of stochastic calculus via regularization". In: Séminaire de Probabilités XL. Vol. 1899. Lecture Notes in Math. Berlin: Springer, 2007, pp. 147-185.

[25] Russo, F. and Vallois, P. "Forward, backward and symmetric stochastic integration". In: Probab. Theory Related Fields 97.3 (1993), pp. 403-421.

[26] Seignourel, P. "Processus dans un milieu irrégulier. Une approche par les formes de Dirichlet." In: Preprint RI, 388, CMAP-Ecole Polytechnique (1998). 
[27] Stroock, D. W. and Varadhan, S. R. S. Multidimensional diffusion processes. Classics in Mathematics. Reprint of the 1997 edition. Berlin: Springer-Verlag, 2006, pp. xii +338 .

[28] Szarski, J. Differential inequalities. Monografie Matematyczne, Tom 43. Państwowe Wydawnictwo Naukowe, Warsaw, 1965, p. 256. 